\section{(6) OPEN ACCESS}

\title{
Polarised epithelial monolayers of the gastric mucosa reveal insights into mucosal homeostasis and defence against infection
}

\author{
Francesco Boccellato, ${ }^{1}$ Sarah Woelffling, ${ }_{1}^{1}$ Aki Imai-Matsushima, ${ }^{1}$ Gabriela Sanchez, ${ }^{1}$ \\ Christian Goosmann, ${ }^{1}$ Monika Schmid, ${ }^{1}$ Hilmar Berger, ${ }^{1}$ Pau Morey, ${ }^{1}$ \\ Christian Denecke, ${ }^{2}$ Juergen Ordemann, ${ }^{3}$ Thomas F Meyer ${ }^{1}$
}

\begin{abstract}
Additional material is published online only. To view please visit the journal online (http://dx.doi.org/10.1136/ gutjnl-2017-314540).

1 Department of Molecular Biology, Max Planck Institute for Infection Biology, Berlin, Germany

${ }^{2}$ Center for Bariatric and Metabolic Surgery, Charité University Medicine, Berlin, Germany

${ }^{3}$ Department of Bariatric and Metabolic Surgery, Helios Clinics, Berlin, Germany
\end{abstract}

\section{Correspondence to} Professor Thomas F Meyer, Department of Molecular Biology, Max Planck Institute for Infection Biology, Berlin 10117, Germany;

meyer@mpiib-berlin.mpg.de

Received 24 May 2017 Revised 30 January 2018 Accepted 31 January 2018 Published Online First 21 February 2018

\begin{abstract}
Objective Helicobacter pylori causes life-long colonisation of the gastric mucosa, leading to chronic inflammation with increased risk of gastric cancer. Research on the pathogenesis of this infection would strongly benefit from an authentic human in vitro model. Design Antrum-derived gastric glands from surgery specimens served to establish polarised epithelial monolayers via a transient air-liquid interface culture stage to study cross-talk with $H$. pylori and the adjacent stroma.

Results The resulting 'mucosoid cultures', so named because they recapitulate key characteristics of the gastric mucosa, represent normal stem cell-driven cultures that can be passaged for months. These highly polarised columnar epithelial layers encompass the various gastric antral cell types and secrete mucus at the apical surface. By default, they differentiate towards a foveolar, MUC5AC-producing phenotype, whereas Wnt signalling stimulates proliferation of MUC6-producing cells and preserves stemness - reminiscent of the gland base. Stromal cells from the lamina propria secrete Wnt inhibitors, antagonising stem-cell niche signalling and inducing differentiation. On infection with $\mathrm{H}$. pylori, a strong inflammatory response is induced preferentially in the undifferentiated basal cell phenotype. Infection of cultures for several weeks produces foci of viable bacteria and a persistent inflammatory condition, while the secreted mucus establishes a barrier that only few bacteria manage to overcome.

Conclusion Gastric mucosoid cultures faithfully reproduce the features of normal human gastric epithelium, enabling new approaches for investigating the interaction of $H$. pylori with the epithelial surface and the cross-talk with the basolateral stromal compartment. Our observations provide striking insights in the regulatory circuits of inflammation and defence.
\end{abstract}

\section{INTRODUCTION}

Helicobacter pylori, a Gram-negative bacterium that infects around half of the human population, can persist in the stomach over decades and in some cases lead to gastric adenocarcinoma. ${ }^{1-4}$ The exceptional ability to escape the mucosal immune defence appears intrinsically connected with a chronic inflammatory status. ${ }^{5-7}$ The inflammatory response

\section{Significance of this study}

What is already known on this subject?

- The gastric mucosa consists of a columnar epithelial monolayer protected by mucus. This defence barrier can be colonised by Helicobacter pylori, triggering inflammation with an increased risk of gastric cancer.

- Recent years have seen the development of human gastric organoid cultures enabling mechanistic investigations in primary cells.

- Yet, with regard to the assessment of infection processes, plain organoids present suboptimal conditions for infection studies, due to their closed spherical shape, luminal polarisation with accumulating deposition of apoptotic cells in the lumen, as well as their encapsulation into extracellular matrix.

What are the new findings?

-We have regenerated the columnar epithelium of the human gastric mucosa following the approach of an air-liquid interface (ALI) culture. However, in contrast to standard ALI cultures, the gastric polarised epithelial monolayers (mucosoid cultures) are long-lived. Mucosoid cultures constitute stable cultures consisting of highly polarised cells comprised of all the gastric gland lineages. They allow for homeostatic infections, eg, with $H$. pylori, for periods of several weeks.

- Under the influence of Wnt signalling, gastric mucosoid cultures differentiate into phenotypes typical of the base of the gland. Stromal cells secrete Wnt inhibitors and drive differentiation towards a foveolar phenotype. Only basal cells respond to $H$. pylori infection with a strong inflammatory response.

- Gastric mucosoid cultures produce and accumulate abundant mucus on the apical side, which prevents immediate pathogen contact with epithelial cells and controls bacterial proliferation.

of the mucosa mediates the host defence against infection and tissue repair through enhanced regeneration, an aspect that has been implicated in tumour development. ${ }^{8}$ 
Significance of this study

\section{How might it impact on clinical practice in the foreseeable future?}

- The gastric mucosoid culture model will generate new insights into the effects of long-term infection with $H$. pylori on epithelial cells, paving the way for new therapeutic approaches against infection and its sequelae.

- The gastric mucosoid culture model is likely to be transferrable to other columnar epithelia. It promises to be a versatile drug-screening tool, enabling compound testing with differential applications on either or both the apical and the basal sides. It also allows assessment of molecular communication in co-culture with other cell types, such as myofibroblasts and immune cells.

- The robust mucus production will enable insights into the protective role of this important mucosal component and provide new options for future therapeutic drug targeting.

H. pylori adheres to the gastric foveolae, ${ }^{9}$ but it can eventually infiltrate the glands ${ }^{10}$ and interact with the stem cell compartment located at the base. ${ }^{11}$ Experiments in mice have revealed that hyperplasia and other pathological changes in the stomach occur mainly in response to such deep glandular colonisation. ${ }^{11}$ More detailed investigations into the infection dynamics therefore would benefit from a robust in vitro model that recapitulates the features of the basal glandular compartment.

In recent years, an increased understanding of stem cell biology has driven the development of organoid cultures as a way of maintaining adult stem cells in vitro, ${ }^{12} 13$ including those obtained by isolating glands from gastric tissue samples. ${ }^{14} 15$ The epithelium of the antrum is maintained by $\operatorname{Lgr} 5+$ stem cells located at the base of the glands ${ }^{16}$ where MUC6 is secreted. Maintenance of these stem cells in vitro via the niche signalling factors Wnt and R-spondin enables long-lived cultures of gastric organoids, and it has been hypothesised that the stroma plays a crucial role in this via secretion of niche factors. ${ }^{17-19}$

Organoids can be injected with $H$. pylori to model infection in vitro, ${ }^{20}{ }^{21}$ but this system does not support large-scale infection studies. An alternative approach has been to transfer organoid cells to two-dimensional (2D) monolayer culture, ${ }^{14}$ but infected cultures succumb within 24 hours. This may be due not only to the loss of tissue architecture and polarisation, enabling bacterial access to a much larger fraction of the cell surface than in situ but also to the dispersal of the protective mucins. A system that enables routine infection while maintaining the epithelial architecture characterised by polarised cells and tight junctions, as well as an intact mucus layer, may thus support long-term infection with $H$. pylori.

Here, we harnessed air-liquid interface (ALI) methodology to establish 'mucosoid' cultures-functional, long-lived, polarised epithelial monolayers-from healthy human stomach tissue, which fully recapitulate the different cell lineages found in antral gastric glands in situ. These advances were obtained by combining established cultivation methods based on polycarbonate filters ${ }^{18} 22$ with more recent knowledge about propagation of primary GI cells from adult stem cells. ${ }^{14} 1523$

We further show that this system enables directed differentiation into foveolar or basal phenotypes by either Wnt signalling modulation or coculturing with a defined stromal population. The apical surface of the monolayer is protected by secreted mucus, enabling chronic in vitro infection with $H$. pylori. These features represent key advantages over existing culture methods and permit advanced studies on the mechanisms of chronic inflammation, the role of mucus in epithelial defence and the factors that control epithelial homeostasis during infection. As such, it represents an important new tool for illuminating the mechanisms underlying gastric carcinogenesis.

\section{RESULTS}

\section{Establishment of gastric mucosoid cultures on filters}

Gastric glands were isolated from human antrum sleeve gastrectomy samples (figure 1A) as previously described. ${ }^{14}$ Antrum (figure 1A dotted line) was identified as the region opposite to the fundus (marked by the surgeon) that does not contain rugae typical of the corpus. Dissociated cells from fresh tissue or gastric organoids were seeded onto collagen-coated polycarbonate filters. After 3 days, the medium on top of the cells is removed to initiate air-liquid interface conditions (figure 1B). Over the next 10 days, they develop a mature epithelial morphology, becoming progressively taller (figure 1C). After 10-13 days, transparent mucus starts to accumulate on top of the monolayer $(\sim 50-100 \mu \mathrm{L} /$ week) and has to be removed regularly (see online supplementary movie). Cells from mature monolayers can be passaged onto new filters or grown as organoids and vice versa, thus the two methods are interconvertible. Gastric mucosoid cultures contain sporadic Ki67-positive proliferative cells (figure 1D), even after 1 and 2 months of culture (figure 1E,F), indicating that they are stable even without passaging.

We used quantitative reverse transcription PCR (qRT-PCR) to monitor the long-term stability of cultures that were passaged once a month. Expression of the gastric epithelial markers KRT8, 18 and 19, EPCAM and CHD1 remained constant for up to 7 months (figure 1G). Expression of the gastric stem cell marker $\operatorname{Lgr} 5^{12}$ also remained constant after the first passage, as did $\beta$-catenin and $C D 44$, a marker of proliferating and stem cells in the stomach ${ }^{24}$ (figure $1 \mathrm{H}$ ). At the same time, markers of differentiated gland phenotypes were also expressed at constant levels, including pepsinogen $\mathrm{C}(P G C)$ for chief cells, MUC6 for cells at the gland base, MUC5AC for foveolar, chromogranin A (CHGA) for enteroendocrine and ATP4B for parietal cells (figure 1I), indicating that the phenotypic composition remains unchanged over time.

\section{Prominent polarisation of mucosoid cultures}

The polarisation of gastric mucosoid cultures reflects that observed in the stomach, with the apical side facing up, where mucus is accumulating and the basal side facing the filter, where nutrients and growth factors are taken up. Nuclei are localised on the basal side (figure 2A), while E-cadherin is expressed only basolaterally and never apically (figures 1C and 3D postseeding) and expression of the tight junction marker occludin is restricted to the apical side (figure 2B). Transepithelial electrical resistance (TEER) to ion migration ranged from 370 to $470 \Omega * \mathrm{~cm}^{2}$, depending on cell density, which is higher than colon but lower than lung epithelium. ${ }^{25} \mathrm{~A}$ top view shows that tight junctions (green) appear as characteristic dots connecting apically contiguous cells, forming a continuous barrier (figure 2C). Since epithelial barriers have a high turnover, proliferation as well as cell extrusion are equally important to preserve constant cell numbers. Interestingly, when we imaged lower stacks, we found invaginations of tight junctions that formed rosette-like structures with the surrounding cells (figure 2D). A similar phenomenon has been described during cell extrusion, which triggers basal physiological rearrangement of tight junctions ${ }^{2627}$ 
A

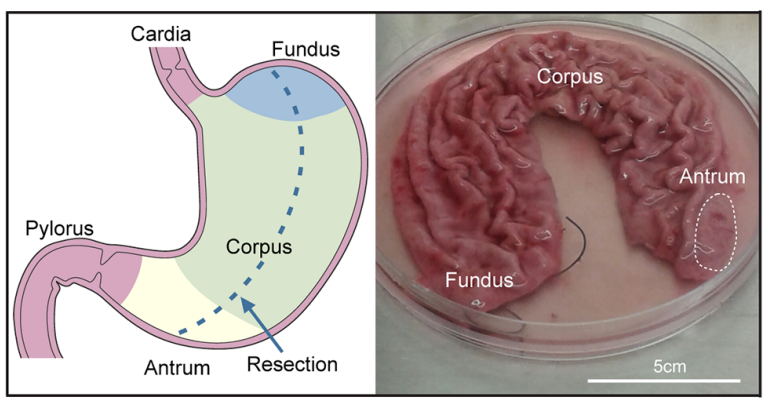

C
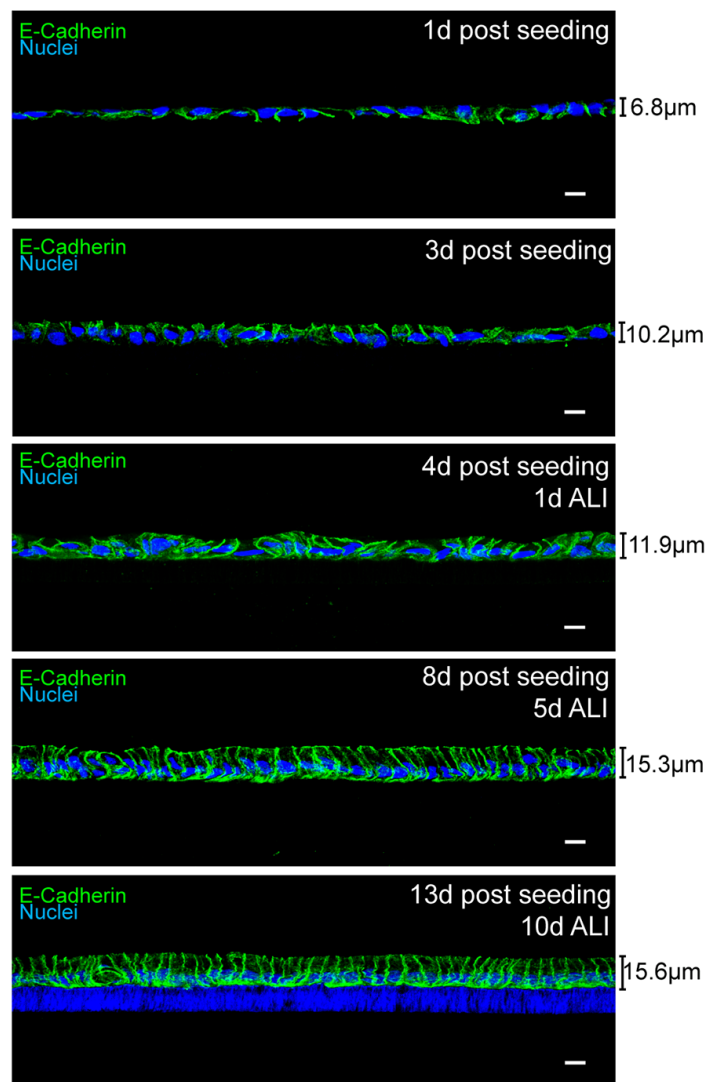

H

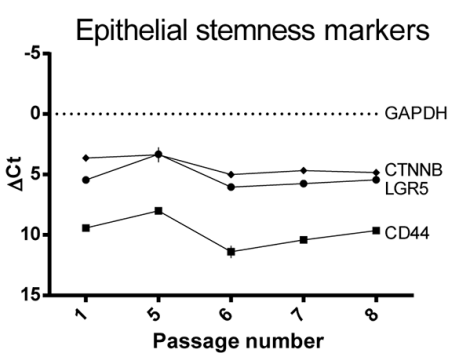

B

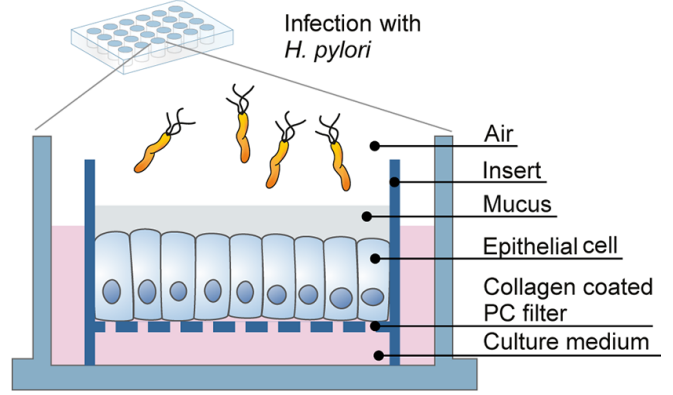

D

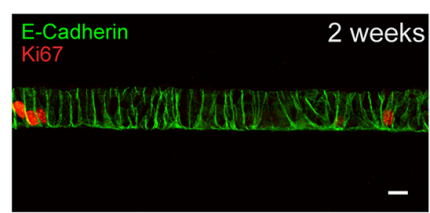

E

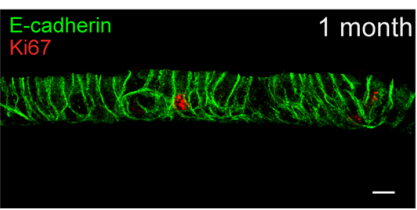

$\mathbf{F}$

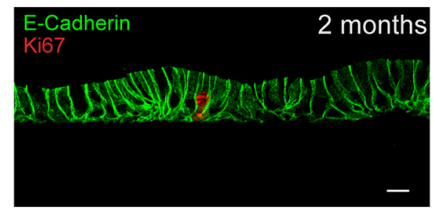

G
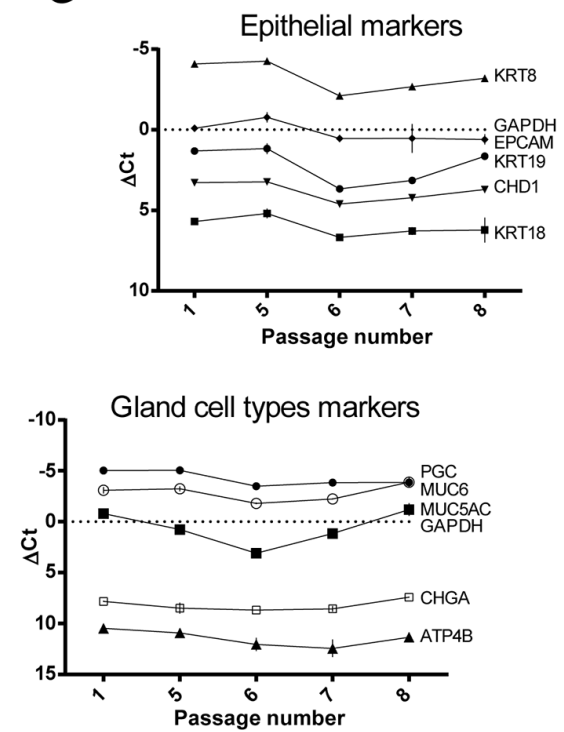

Figure 1 (A) An example of sleeve resection showing the location of antrum, corpus and fundus. The fundus (marked by the surgeon) defines the orientation of the tissue. Antrum is taken from the lower part of the resection devoid of gastric rugae. (B) Schematic of mucosoid culture method: cells are seeded at confluent density onto polycarbonate filters of well inserts placed in a 24-well plate and the medium above the cell layer is withdrawn after 3 days to initiate air-liquid interface culture (C) 1 day after seeding cells have formed a confluent monolayer. On day 3, they have increased in height and medium above the cells is removed. The height and polarisation of the cell layer continues to increase, and on day 10, a layer of mucin is present on the surface. Monolayer height was measured using FIJ software. (D-F) Immunofluoresence (IF) labelling with Ki67 antibody marks proliferating cells in mucosoid cultures between 2 weeks and 2 months of culture. (G-I) mRNA expression levels of genes specific for epithelial (G), stem (H) and gland cells (I) determined from two parallel mucosoid cultures over eight passages (6 months), as determined by qPCR. $\triangle \mathrm{Ct}=$ difference between the $\mathrm{Ct}$ of each gene compared with GAPDH. Results are from one of two analysed mucosoid cultures. Scale bars: $10 \mu \mathrm{m}$. GAPDH, glyceraldehyde 3-phosphate dehydrogenase; H. pylori, Helicobacter pylori; mRNA, messenger RNA; qRT-PCR, quantitative reverse transcription PCR. 
A

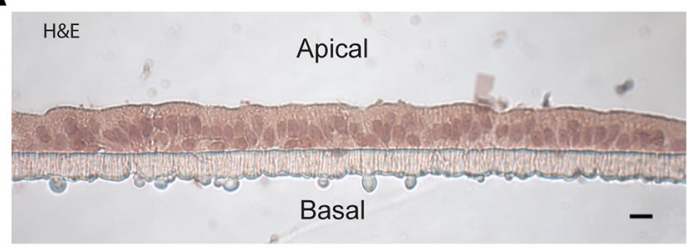

C

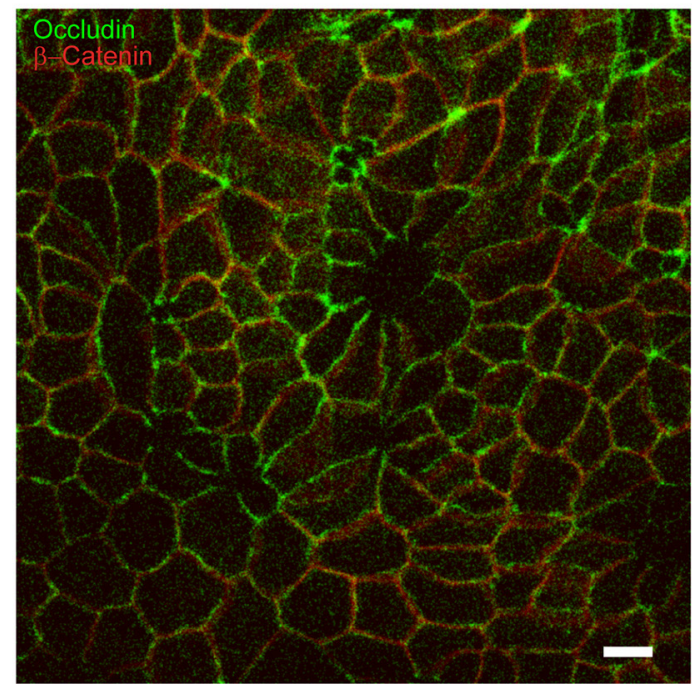

E
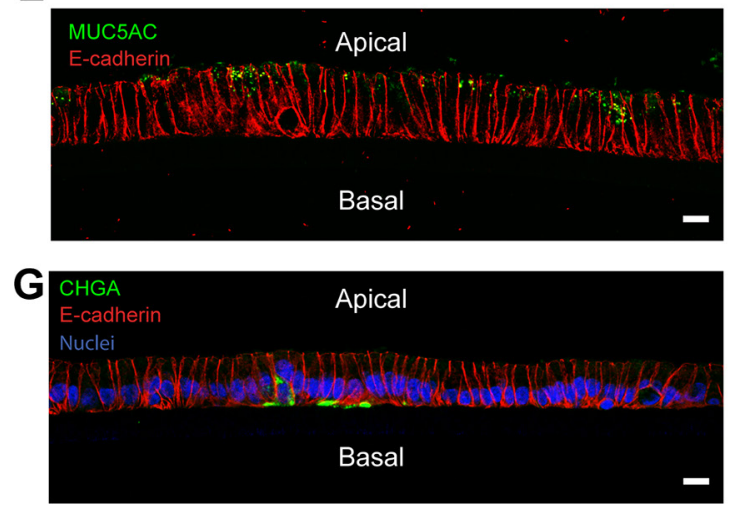

B

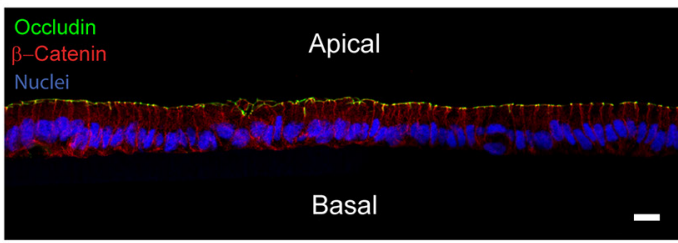

D
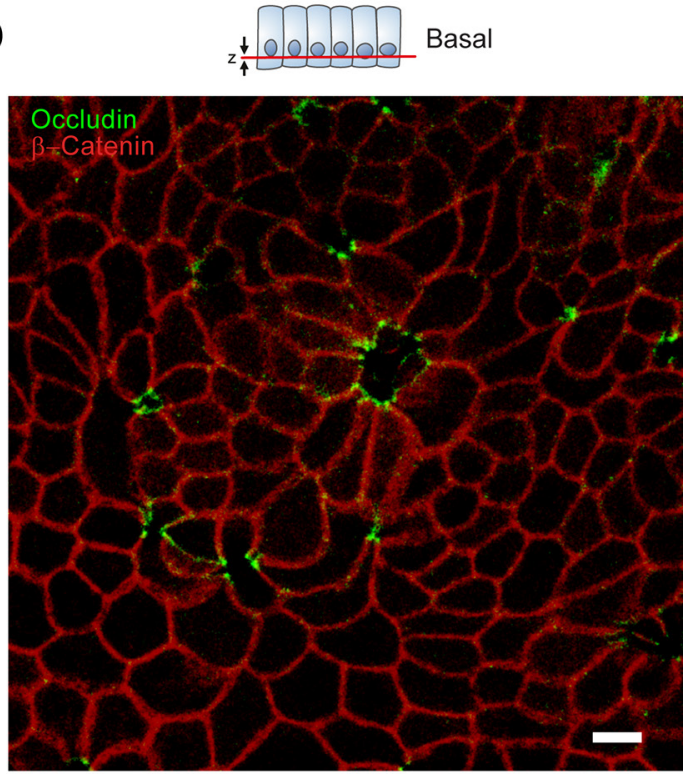

F

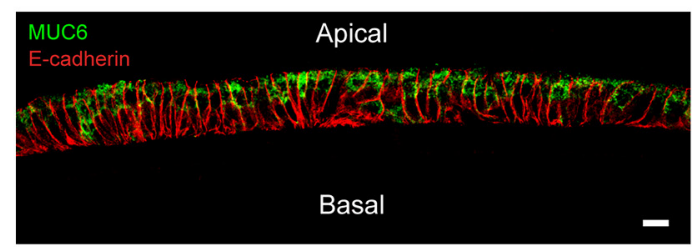

$\mathrm{H}$

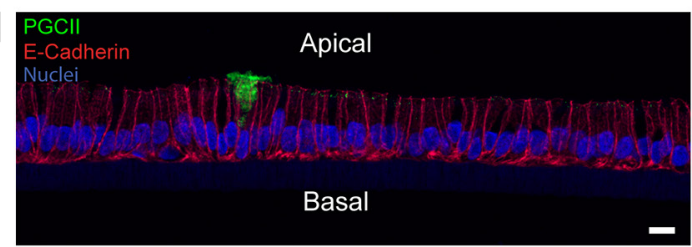

Figure 2 (A) H\&E staining of a mucosoid culture section shows basal distribution of the nuclei. (B) IF labelling against occludin shows that cells are apically connected by tight junctions. (C) IF top view of a mucosoid culture showing that occludin is present in all cell junctions. (D) IF image of a lower section of the same monolayer showing basal rearrangement of apical occludin around cells organised into a rosette-like structure. (E) Few granules in the cells from $(+\mathrm{W}+\mathrm{R})$ antrum-mucosoid cultures are positive for MUC5AC apically. (F) cells from $(+\mathrm{W}+\mathrm{R})$ antrum-mucosoid cultures shows a high number of apical MUC6 positive granules. (G) IF labelling against chromogranin A showing positive granules on the basal side of hormone-producing enteroendocrine cells of an antral mucosoid culture (H). IF labelling against pepsinogen CII showing pepsinogen II-producing chief cells in an antrum-derived mucosoid culture. Scale bars: $10 \mu \mathrm{m}$.

to maintain the epithelial barrier integrity. To further stress the high polarisation of the cultures, we analysed gastric epithelial secreted proteins in the apical and basal compartments. Glands contain large numbers of mucus-producing cells, in particular the mucin MUC5AC. IF analysis of undifferentiated mucosoid cultures also revealed few intracellular MUC5AC and abundant MUC6 granules that accumulate apically for secretion (figure 2E-F) and which contribute to the formation of an apical mucus layer (see online supplementary movie 1 ). On the other hand, we observed basal accumulation of CHGA granules typical of enteroendocrine cells (figure 2G and online supplementary figure 1A). Rare PGCII-positive cells $(<0.5 \%)$ detected in the mucosoid cultures represent chief cells releasing pepsinogen on the apical side (figure $2 \mathrm{H}$ and online supplementary figure $1 \mathrm{~B}$ ).

\section{Differentiation and proliferation dynamics}

Lineage tracing experiments in mice have demonstrated that the base of antral glands is populated by $\operatorname{Lgr} 5$-expressing stem cells that are able to repopulate the whole gland. These cells proliferate and migrate up to the pit of the gland, forming the foveolar lineage. ${ }^{12}$ The stem cell niche in vivo largely depends 
A

C
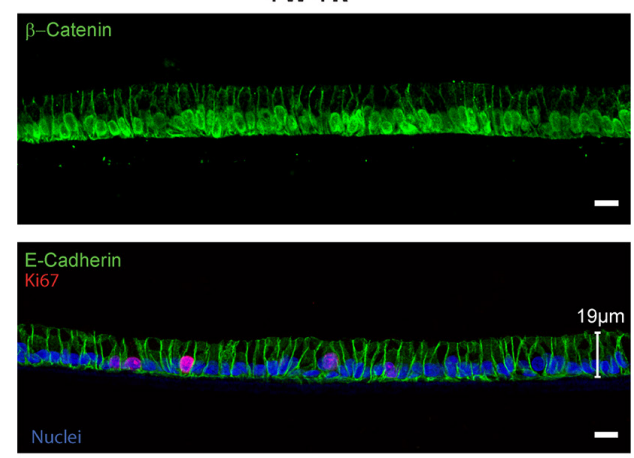

E

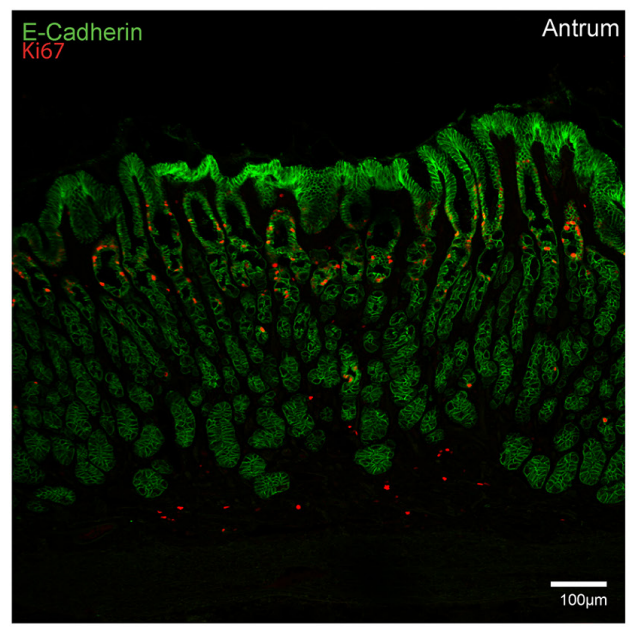

B
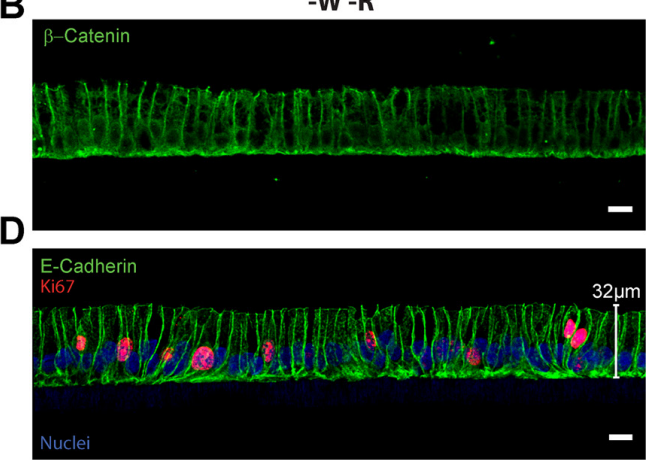

$\mathbf{F}$

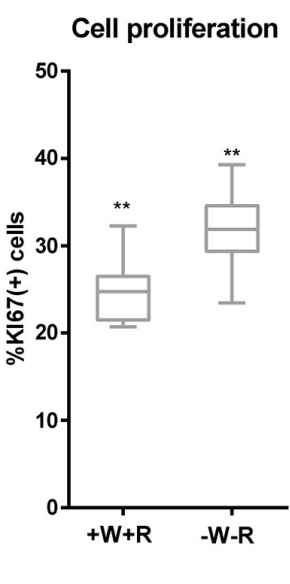

G

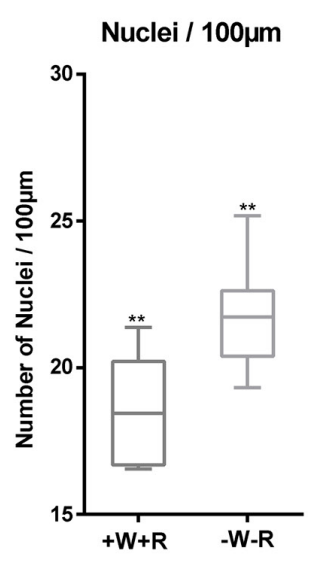

H
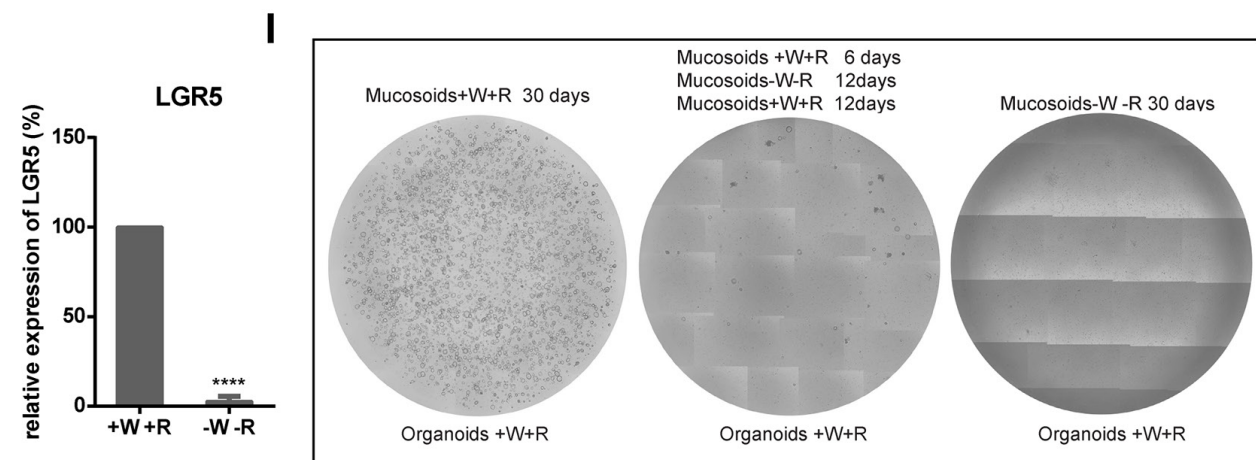

Figure 3 Two or three distinct antrum mucosoid cultures were used to evaluate the effect of Wnt and RSPO on cellular differentiation and proliferation. (A) IF labelling against $\beta$-catenin showing universal nuclear localisation in $+W+R$ condition. (B) $\beta$-catenin is absent from the nucleus after W/R are withdrawn for 6 days. (C,D) Withdrawal of W/R is also accompanied by an increased number of cells positive for the proliferation marker Ki67 and an increase in height. (E) Stomach antrum section stained for Ki67 shows the subfoveolar localisation of the proliferation compartment in the antrum. $(F, G)$ Quantification of the Ki67 positive cells and of the the cell density after withdrawal of W/R from eight sections $n>400$ (H) LGR5 determined by qRT-PCR relative to that in $+W+R$ medium. Bars represent the mean of three. (I) Tile scans of a $50 \mu l$ Matrigel drop seeded with 30000 cells followed by 6 days of spheroid growth. Left: mucosoid cultures were cultured for 30 days in $+W+R$ medium before passaging into Matrigel cultures in $+W+R$ medium to grow organoids. Shown is a representative images of three independent cultures. Centre: Mucosoid cultured for 6 days in $+W+R$ medium followed by removal of Wnt3A and RSP01 for 12 days, followed by a further 12 days in $+W+R$ medium leads to a drastic reduction of organoid survival. Right: cells cultured without Wnt3A and RSP01 did not generate any organoids. Unpaired t-test: ${ }^{* * * *} P \leq 0.00005$; ${ }^{* *} P \leq 0.005$. Non-labelled bars: $10 \mu \mathrm{m}$. Lgr5, leucine-rich repeat-containing G-protein coupled receptor 5; qRT-PCR, quantitative reverse transcription PCR; RSPO, R-spondin.

on activation of the Wnt pathway, which is mimicked by supplementing the culture medium with recombinant Wnt3A $(+\mathrm{W})$ and $\mathrm{R}-$ spondin $(\mathrm{RSPO}) 1(+\mathrm{R})^{14}$ leading to the release of $\beta$-catenin in the cytoplasm and translocation into the nucleus (figure $3 \mathrm{~A}$ ), where it acts as a transcriptional coactivator of transcription factors. Depriving cultures of Wnt3A/RSPO1 (-W-R) for 6 days removes $\beta$-catenin from the nucleus (figure $3 \mathrm{~B}$ ) and mimics the process of differentiation into the foveolar phenotype. A proliferative zone in the stomach is found just below the pits of the gastric mucosa. In the antrum, which is characterised by a higher foveolar compartment, this zone is located deep in the pits ${ }^{28}$ (figure 3E). In cultures deprived of Wnt3A and 
RSPO1, we noticed an elevated number of Ki67+ proliferative cells (figure 3C,D quantification in F), which increases the height and the density of cells, which is typical of the pits (figure $3 \mathrm{G}$ ). Deprivation of Wnt3A and RSPO1 further resulted in a drastic reduction in the expression of the stem cell marker LGR5 (figure $3 \mathrm{H}$ ). To test whether this differentiation is reversible, we seeded primary cells on filters to establish mucosoid cultures and then withdrew Wnt3A and RSPO1 for 12 or 30 days. Single cells were transferred to organoid culture conditions, restoring the supply of WN3A and RSPO1. The ability to form organoids was greatly and permanently reduced compared with controls, even when Wnt and RSPO were only withdrawn for 12 days (figure 3I).

\section{Differentiation regulates mucus secretion}

The glands of the gastric mucosa are protected against acid and digestive enzymes by a mucus gel that polymerises at different densities. In the normal mucosa of the antrum, MUC5AC is produced on the surface, while MUC6 is expressed deeper in the gland..$^{29}$ Similarly, mucosoid cultures grown in $+\mathrm{W}+\mathrm{R}$ medium contain low levels of MUC5AC (red) (figure 4A), while -W-R medium induces extensive production of this mucin (figure 4B). The opposite is observed for MUC6 (figure 4C,D). A mixed MUC5AC/MUC6 as well as a mild proliferative phenotype is obtained if Wnt3A and RSPO1 are removed alternatively (see online supplementary figure 2 ). The mucin granules are detectable by electron microscopy and accumulate on the apical side in both conditions, although in the -W-R cultures, the apical granules are larger and more numerous (figure 4E,F). This differential production of mucins was confirmed by RT-PCR (figure 4G). Mass spectrometry analysis of mucus from $+W+R$ cultures compared with -W-R cultures confirmed that the most abundant mucins are MUC5AC and MUC6 and that the latter is secreted in $+\mathrm{W}+\mathrm{R}$ cultures, representing basal conditions (figure $4 \mathrm{H}$ and online supplementary table 1). Trefoil factors, trypsin 1 and 2, gastrokine 1 and especially the antimicrobial protein lysozyme are also abundant in the mucus (figure $4 \mathrm{H}$ and online supplementary table 1). Interestingly, the stem cell-related protein PROM1 was also present at high levels in the mucus, although its secretion is only predicted (figure $4 \mathrm{H}$ ). Mucosoid cultures thus recapitulate the main features of the original tissue and offer a unique opportunity to study the mucus and the changes in the apical secretome under different physiological and eventually pathological conditions.

\section{Cross-talk between stromal cells and epithelium}

The gastric mucosa is organised into glands intercalated by the lamina propria, which consists of stromal fibroblasts. These stromal cells differ from the myofibroblast of the lamina muscularis mucosae directly beneath the glands (figure $5 \mathrm{~A}$ ). We isolated and expanded gastric stromal cells (GSCs) of the lamina propria and added them to the basal compartment of the cultures. After 6 days, the level of epithelial LGR5 messenger RNA (mRNA) was reduced, as determined by q-PCR. This effect was reproduced by exposing cultures to GSC-conditioned medium (figure 5B), suggesting that a secreted factor is responsible. To test if the reduction in Lgr5 expression was due to alterations of the Wnt-signalling pathway, we titrated the GSC-conditioned medium on a Wnt reporter cell line activated with $50 \%$ of LWnt3A supernatant (a cell line producing abundant Wnt3A) (figure $5 \mathrm{C}$ ). While $50 \% \mathrm{LWnt} 3 \mathrm{~A}$ supernatant generated a strong reporter signal (figure 5C and online supplementary figure 3A upper panel), combination with GSC-conditioned medium reduced the signal (figure 5C and online supplementary figure 3A lower panel). Distribution of $\beta$-catenin also showed nuclear location only when the Wnt pathway is activated by Wnt3A and RSPO1 (figure 5D,F) but not when cells are cocultured with GSCs (figure $5 \mathrm{H}$ ) and undergo partial differentiation into foveolar phenotype, as shown by an increase in MUC5AC producing cells (figure 5I compared with 5G). The stroma of the lamina propria thus reduces the stemness capacity of the epithelium and induces partial differentiation via secreted factors that inhibit the Wnt pathway in a $\beta$-catenin-dependent fashion. To further analyse which factors are responsible for the reduction in Lgr5 expression, we tested mRNA expression of all the soluble Wnt inhibitors. We observed strong expression of sFRP1, DKK1 and $D K K 3$, as well as BARX1, a transcription factor-regulating $s F R P s$ (figure $5 \mathrm{~J}$ ). As epithelial homeostasis is tightly controlled, signals derived from other cells might perturb this equilibrium, as indicated by the rosette-like structures observed in gastric mucosoid cultures, which indicate possible sites of cell extrusion and regeneration. Rosettes were abundant in cultures containing Wnt activators but almost absent without Wnt activators, while the presence GSCs led to reduced numbers (see online supplementary figure $3 \mathrm{C}-\mathrm{E}$ and quantification in $\mathrm{B}$ ). This indicates that gastric mucosoid cultures enable modelling of epitheliumstroma communication in cocultures, representing a sensitive system for analysing stemness and epithelial homeostasis.

\section{Distinct responses of basal versus foveolar cells to $\boldsymbol{H}$. pylori}

To model infection with $H$. pylori, we infected gastric mucosoid cultures with P12 at multiplicity of infection (MOI) 100 for 3 days. To explore how 'basal' and 'foveolar' cultures respond to infection, mucosoid cultures from the antrum of three patients were cultured for 13 days before withdrawing $\mathrm{Wnt} 3 \mathrm{~A}$ and RSPO1 from half of the cultures for 5 days. The resulting undifferentiated MUC6 ${ }^{\text {hi }}$ and foveolar type MUC5 $\mathrm{AC}^{\text {hi }}$ monolayers were infected for 3 days, then analysed by qPCR and microarray. No significant changes were observed in the viability of bacteria under $+\mathrm{W}+\mathrm{R}$ vs $-\mathrm{W}-\mathrm{R}$ condition (figure $6 \mathrm{~A}$ ). Expression of stem cell-related genes was reduced in differentiated cells, as expected, and expression of MUC6 and MUC5AC changed accordingly (see online supplementary figure 2A,B).

We next focused on the NF-KB-mediated inflammatory response. Upon infection NFKBIA is normally degraded, allowing p65 to enter the nucleus and activate transcription of $\mathrm{\kappa B}$-responsive genes, including I $\mathrm{K} B \alpha$ itself, generating a negative feedback loop. Interestingly, transcription of NFKBIA was much higher if infection was performed in the $+\mathrm{W}+\mathrm{R}$ condition compared with -W-R (figure 6B), suggesting that the ability of the basal compartment to sense bacteria is considerably higher compared with the foveolae. To confirm this observation, we analysed all potential $\mathrm{NF}-\kappa \mathrm{B}$ target genes on our microarray. Following infection, the majority of target genes were upregulated in the $+\mathrm{W}+\mathrm{R}$ compared with the -W-R condition (figure 6C). We used qPCR to validate several important NF- $\mathrm{KB}$-dependent inflammation mediators, including the T-cell-recruiting chemokines interleukin 8 (CXCL8), CXCL1, CXCL3, CCL20 and IL-23A, as well as lymphotoxin B $(L T B)$ (figure 6D-H). Strong upregulation in response to infection was observed only in $+\mathrm{W}+\mathrm{R}$ medium.

As this suggests that cells at the gland base show a much stronger inflammatory response, we tested whether this is due to greater sensitivity to inflammatory stimuli. To this end, $+\mathrm{W}+\mathrm{R}$ and $-\mathrm{W}-\mathrm{R}$ cultures were treated with either tumour necrosis factor alpha (TNF- $\alpha$ ) or IL- $1 \beta$ for 3 days, followed by qPCR. TNF- $\alpha$ induced $I L-8$ expression, regardless of $\mathrm{W} / \mathrm{R}$ (figure $6 \mathrm{~J}$ ), 
A

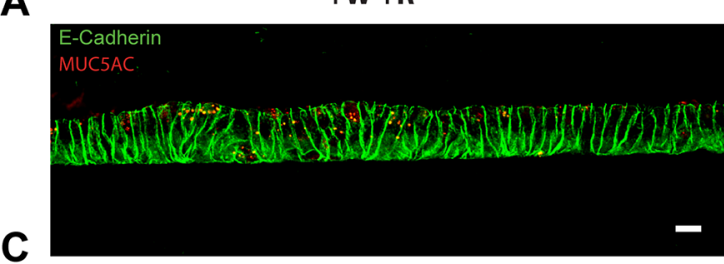

C

E
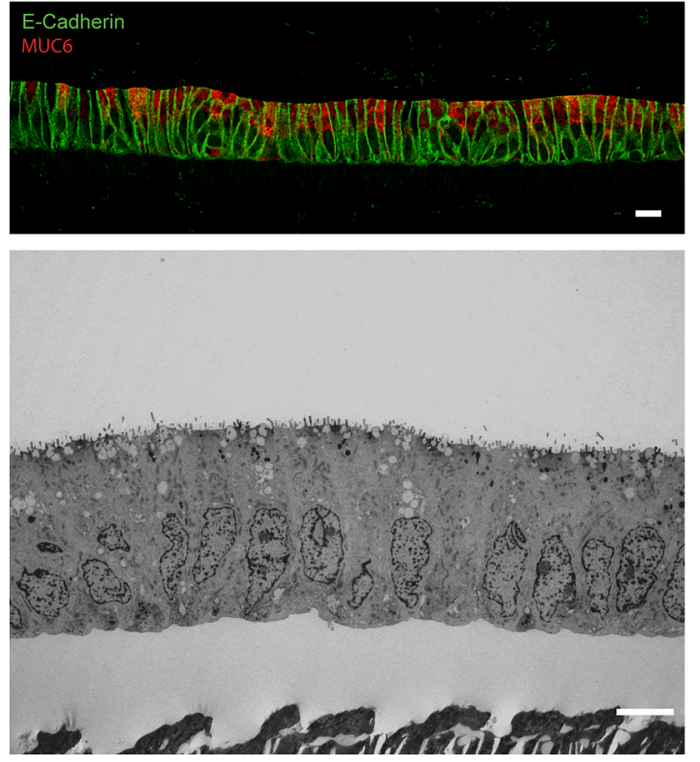

B
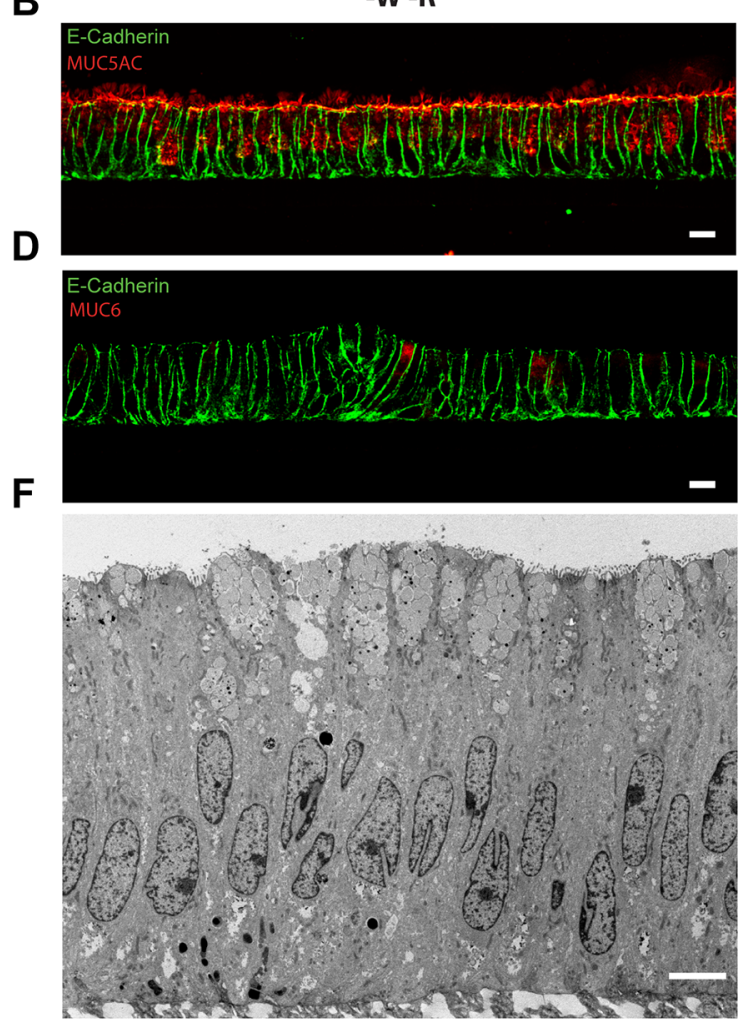

G
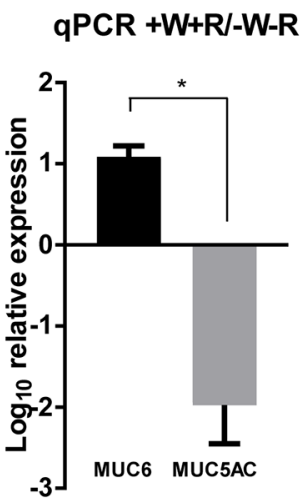

$\mathrm{H}$

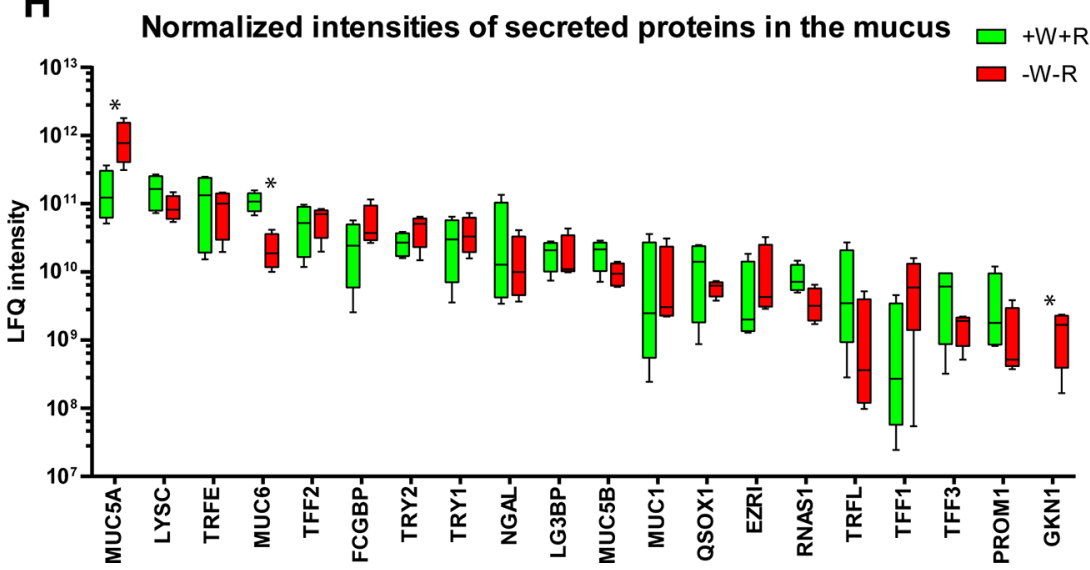

Figure 4 (A-B) IF labelling against the foveolar marker MUC5AC shows that expression is low in the $+\mathrm{W}+\mathrm{R}$ condition and increases after W/R are withdrawn for 6 days (-W-R). (C-D) In contrast, expression of the basal marker MUC6 is high in the $+W+R$ condition and reduces dramatically after W/R are withdrawn for 6 days (-W-R). (E) EM of antral mucosoid culture under $+W+R$ condition showing MUC6-enriched apical mucus granules. (F) Higher accumulation and secretion of MUC5AC-enriched granules in antral mucosoid cultures under -W-R condition. (G) qRT-PCR analysis shows that relative mRNA levels of MUC5AC are higher on W/R withdrawal, whereas levels of MUC6 are high in $+\mathrm{W}+\mathrm{R}$. Bars represent mean $\pm S D$ of two independent experiments ${ }^{*} P<0.05$. (H) Proteome analysis of the total mucus produced by four antral mucosoid culture samples revealed differential secretion of mucins, secreted enzymes and other proteins comparing $+W+R$ vs $-W-R$. Wnt and RSPO were removed for 5 days. Box and whiskers plot represents the average and min/max of normalised LFQ signal calculated with MaxQuant software (four mucus samples per condition). Only highly secreted proteins with more than two unique peptides in the four conditions, with the highest normalised intensity and highest score (>250) and detected in mucus derived from all four antral mucosoid cultures are shown in the graph. EM, electron micrograph; LFQ, label-free quantification; mRNA, messenger RNA; qRT-PCR, quantitative reverse transcription PCR; RSPO, R-spondin.

while IL-1 $\beta$ induced $I L-8$ preferentially in $+\mathrm{W}+\mathrm{R}$ cultures (figure $6 \mathrm{~K}$ ). Since TNF- $\alpha$ but not IL- $1 \beta$ is expressed by epithelial cells, we tested its expression after infection. H. pylori induces expression of TNF- $\alpha$ preferentially in $+\mathrm{W}+\mathrm{R}$ cultures (figure 6I), supporting our observation that the gastric stem cell compartment preferentially responds to infection, while cells in the foveolar compartment remain silent.

\section{Apical dynamics of $\boldsymbol{H}$. pylori infection}

During infection, bacteria were always organised in foci and did not disrupt the monolayer as in conventional 2D culture. We therefore investigated adhesion dynamics and the role of the mucus in controlling the infection. Confocal images of infected antral mucosoid cultures $(+\mathrm{W}+\mathrm{R})$ at 72 hours show some of these colonies still trapped in the mucus (figure 7A left enlargement) 
A

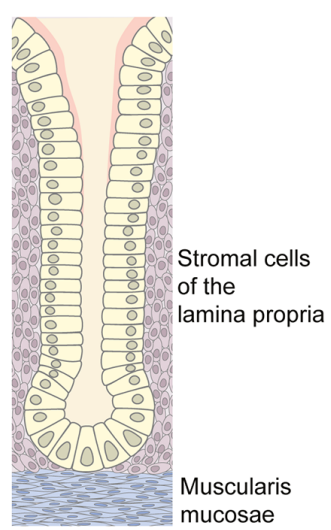

D

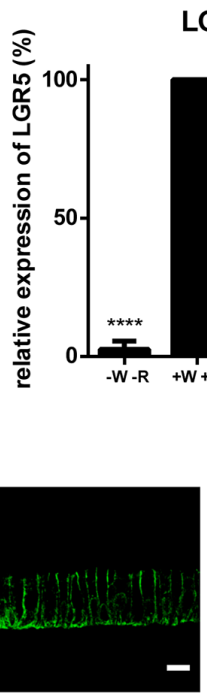

LGR5

$-\mathbf{W}-\mathbf{R}$

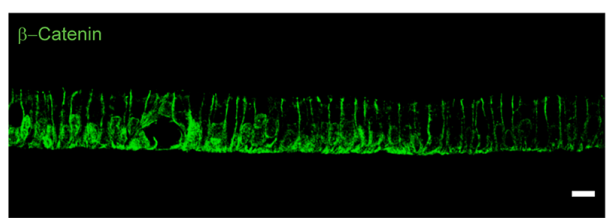

F
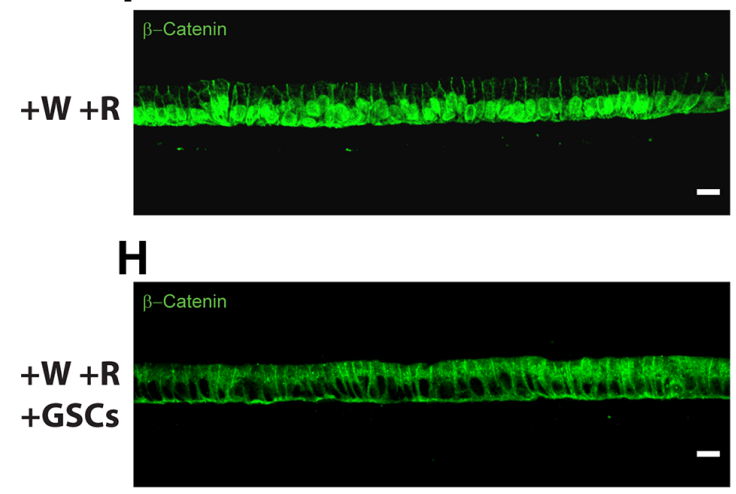

J
C

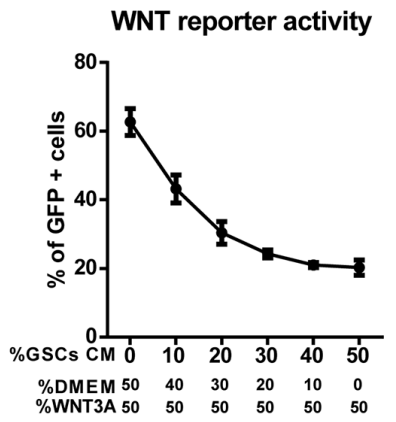

E

Figure 5 (A) Schematic of a gastric gland showing the position of the stroma of the lamina propria from which the isolated GSCs originate. (B) Filters containing antral mucosoid cultures were moved to wells containing GSCs and co-cultured for 6 days, or alternatively cultured with $50 \%$ of identical medium conditioned by GSCs. Levels of $L G R 5$ mRNA expression were measured by qRT-PCR and expressed relative to the $+W+R$ condition; Bars represent mean \pm SEM of three independent biological replicates; ${ }^{*} P<0.05$, ${ }^{* * *}{ }^{*} P<0.00005$, unpaired $t$-test. (C) $293 \mathrm{~T}$ cells transfected with a 7xbinding site for TCF driving GFP expression were used to measure activation of the Wnt pathway. GSC-conditioned medium was titrated in the constant presence of 50\% LWnt3A-conditioned medium. (D, E) Mucosoid cultures grown in -W-R medium show almost no nuclear $\beta$-catenin (D) but highly express MUC5AC (E). (F, G) Antral mucosoid cultures grown in $+W+R$ medium exhibit nuclear $\beta$-catenin (F) but express almost no detectable MUC5AC (G). (H, I) After 6 days of co-culture with GSCs in the presence of $+W+R \beta$-catenin is no longer localised to the nuclei $(H)$ and MUC5AC is expressed again (I). (J) Expression of soluble Wnt inhibitors in GSCS as detected by RT-PCR. Expression of SFRP2 and DKK3 was tested with primers against two different exon-spanning regions. Scale bars: $10 \mu \mathrm{m}$. GSC, gastric stromal cell; mRNA, messenger RNA; qRT-PCR, reverse transcription PCR.

or adhering to the surface of the epithelium (figure $7 \mathrm{~A}$ right enlargement). Translocation of the bacterial CagA protein was confirmed by the presence of phosphorylated protein detected by western blotting (figure 7B). To improve resolution, we used a green fluorescent protein (GFP)-expressing isogenic strain and trimmed the filter to the areas containing green fluorescence to enrich for infected cells before processing for electron microscopy. Interestingly, most of the bacteria are coccoid after
12-24 hours, produce large numbers of vesicles and the majority do not contact the epithelium on the apical surface but rather through the microvilli (figure 7C, left panel). However, helical bacteria can also be found adhering to the surface at 6-hour postinfection (figure 7C, right panel).

Since the apical side is also characterised by the presence of mucus we analysed images of unprocessed sections, which showed that most bacteria are blocked by a layer of mucus (figure 7D), 
A

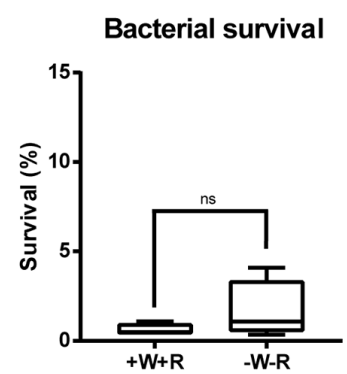

B

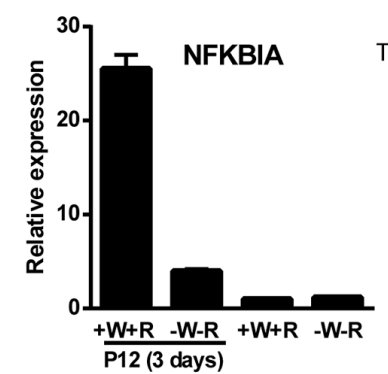

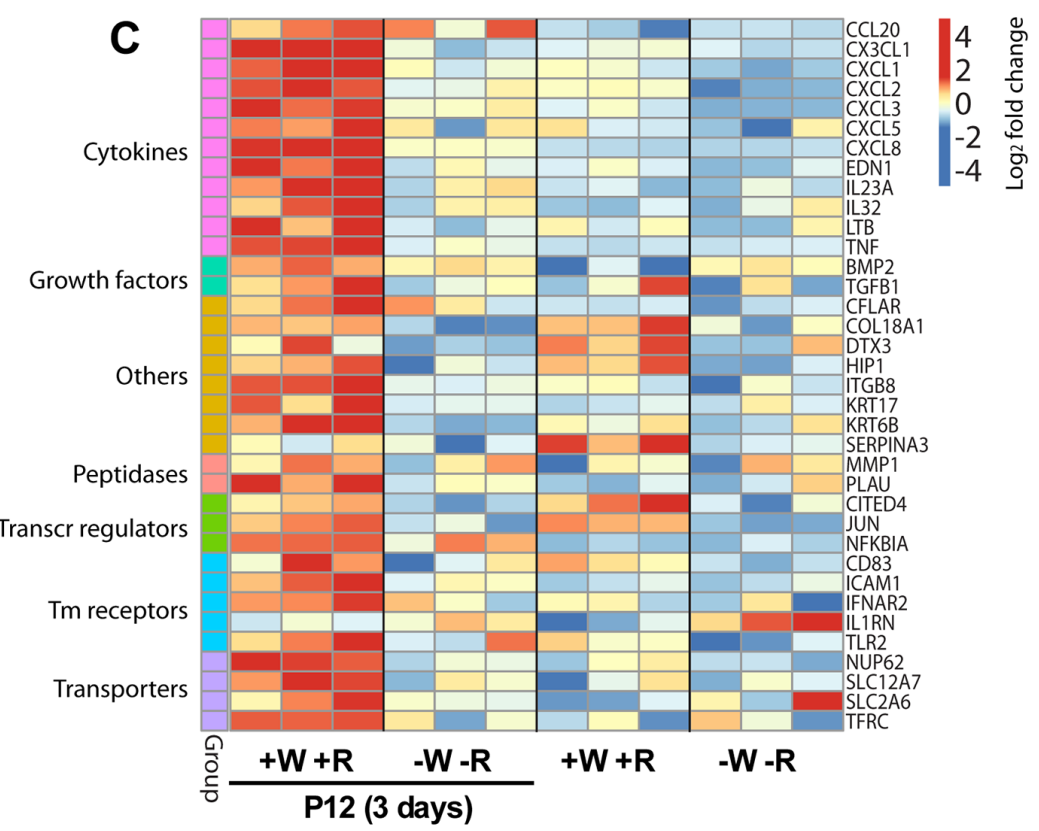

$\mathbf{E}_{10}$
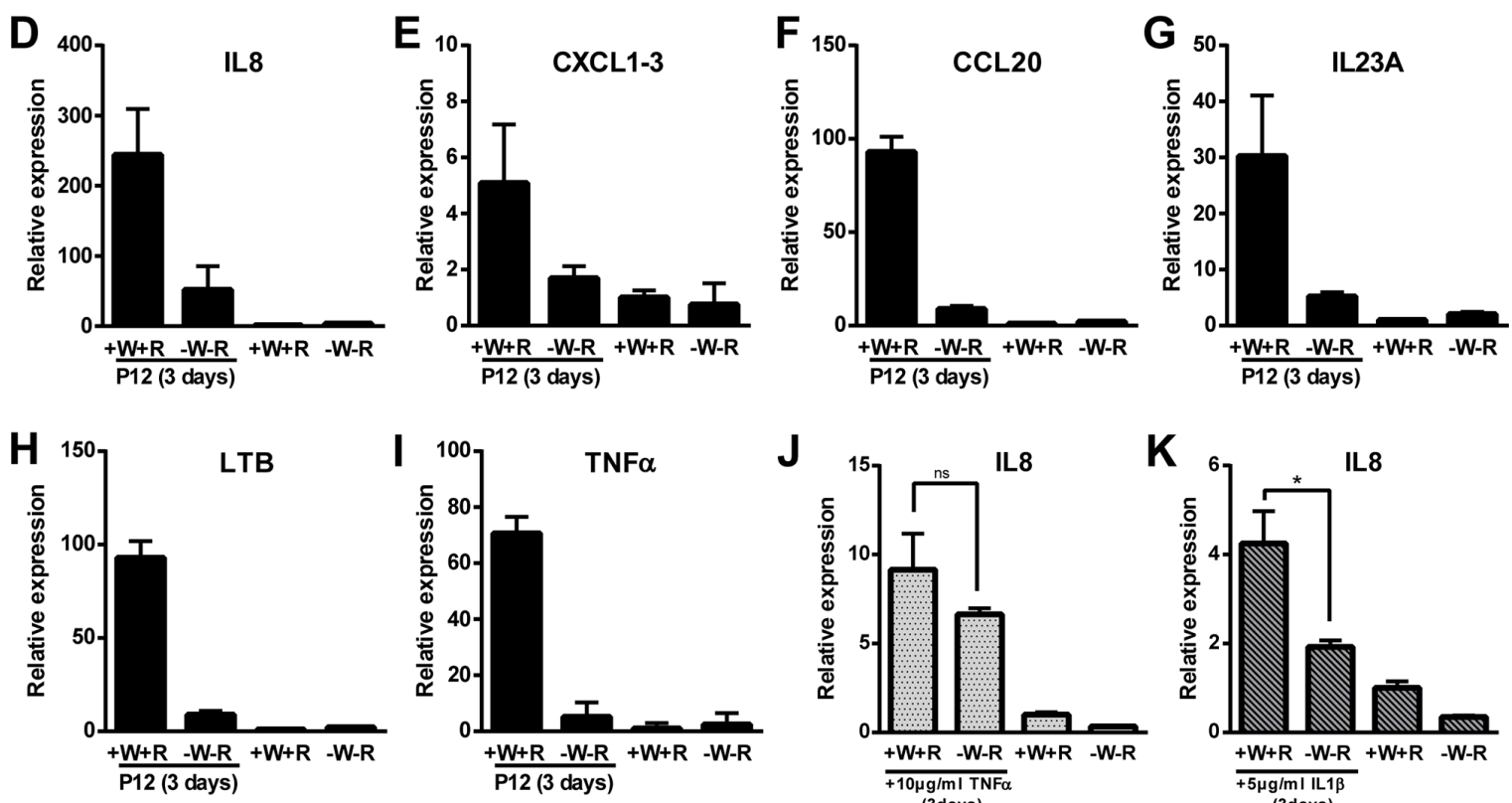
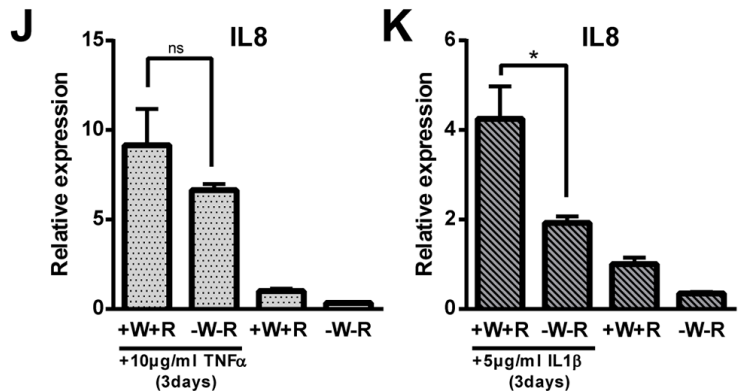

Figure 6 (A) Survival of bacteria (P12) when infecting $+W+R$ antral mucosoid cultures ( $M O I=25)$ compared with $-W-R$ (four different samples per condition) expressed as percentage of input. (B-I) Antral mucosoid cultures derived from three different patients were cultivated for 13 days. Parallel samples were differentiated by excluding Wnt3A and RSP01 from the culture for 5 days, followed by infection with P12 for 3 days at MOI 100. (B) Expression of IKB $\alpha$ (NFKBIA) was analysed by qRT-PCR and normalised to non-infected control cells in $+W+R$. Results shown are representative of two biological replicates. (C) RNA from three biological replicates was analysed by microarray. The heatmap represents NF- $\mathrm{KB}$ target genes grouped by their function. (D-I) Validation of selected array results by qRT-PCR normalised to non-infected control cells in $+W+R$. Results shown are representative of two biological replicates. (J, K) Mucosoid cultures from three different patients were cultured as in (B-I) then treated with $10 \mu \mathrm{g} /$ $\mathrm{mL}$ TNF- $\alpha$ or $5 \mu \mathrm{g} / \mathrm{mL} \mathrm{LL}-1 \beta$ for 3 days. Expression of $I L-8$ was analysed by qRT-PCR and normalised to untreated control cells in $+W+R$ medium. ns: not significant; ${ }^{*} \mathrm{P}<0.005$, unpaired t-test. Error bars represent minimum and maximum values from technical replicates. IL, interleukin; qRT$\mathrm{PCR}$, quantitative reverse transcription PCR; TNF-a, tumour necrosis factor alpha.

which they can traverse only rarely to establish focal colonies on top of the cells (arrowheads). This might explain why the mucosoid cultures are not homogeneously infected. Interestingly, we observed that the mucus is a physical barrier and has bactericidal activity. We aspirated surface mucus from P12-infected and non-infected antral cultures $(+\mathrm{W}+\mathrm{R})$, removed bacteria by centrifugation and incubated with an isogenic P12-GFP strain expressing a kanamycin resistance cassette in the GFP vector, plated at different dilutions on kanamycin plates, then counted colonies. Mucus from infected cultures killed all bacteria after 2 hours, while mucus from uninfected cultures did not impair bacterial viability at all (figure 7E). This shows that the stomach mucus has a central role in controlling $H$. pylori infection, acting first as a physical barrier, which then becomes toxic to the bacteria once the epithelium is infected. This might explain why in our mucosoid cultures as well as in vivo the infection is 
A
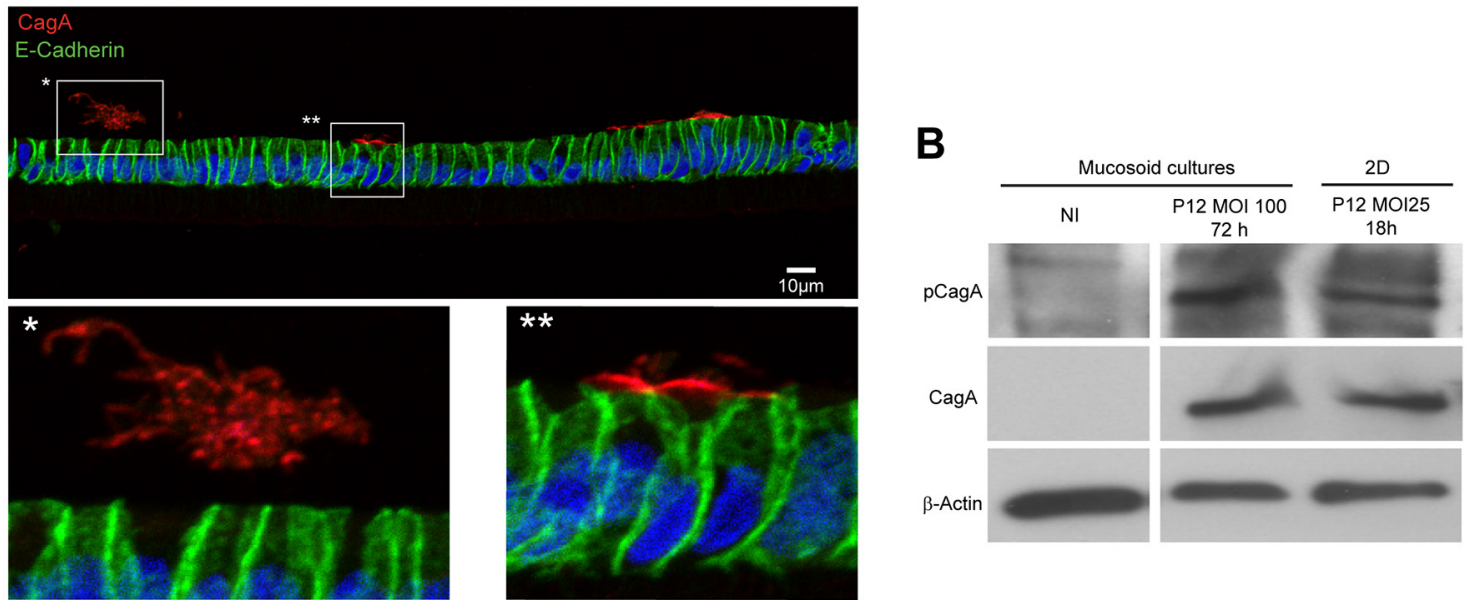

C

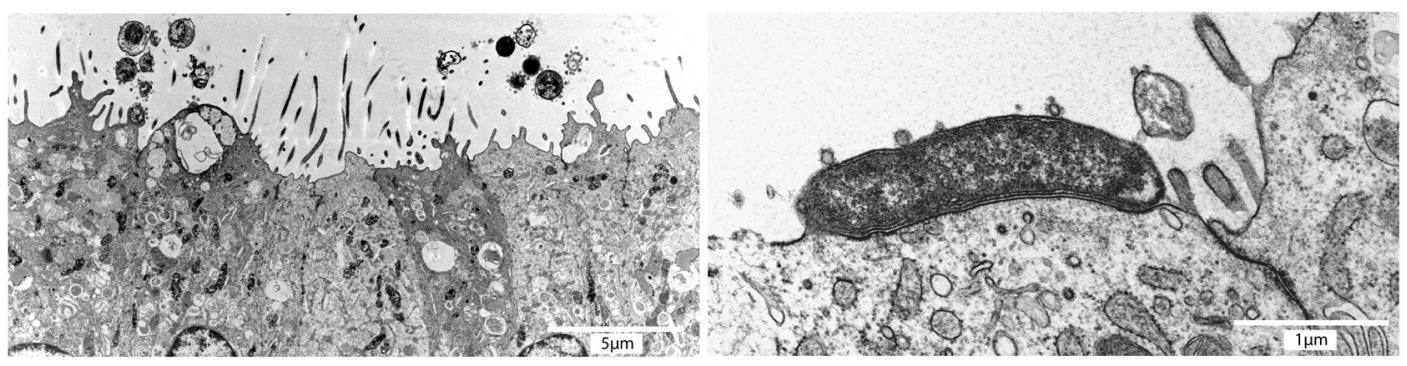

D

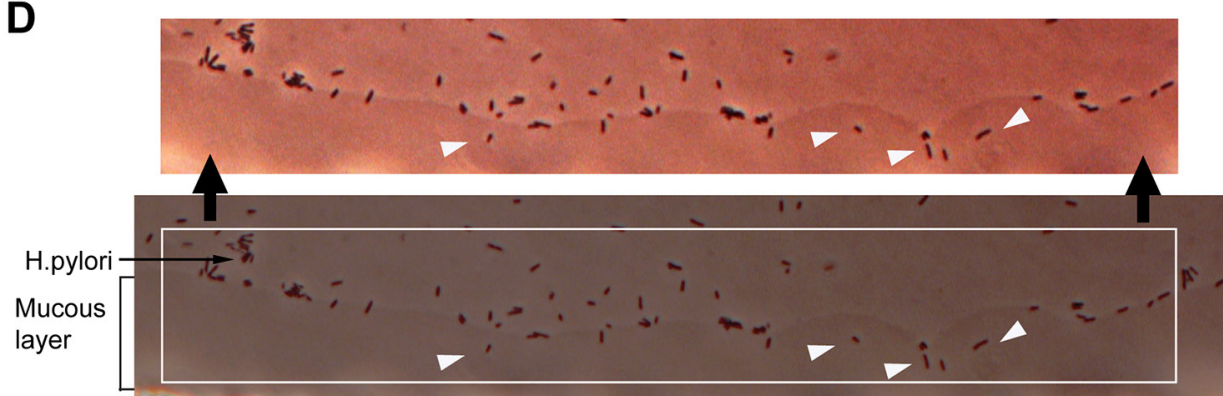

cells+ filter

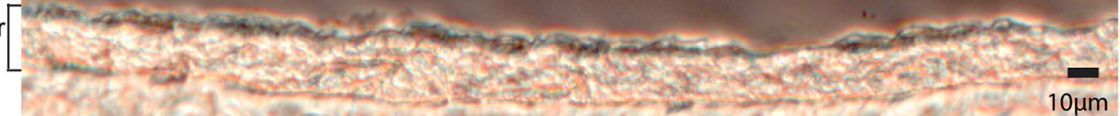

E Mucus

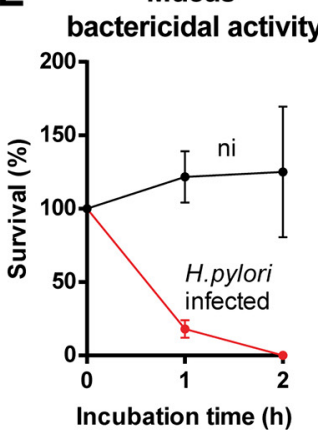

Figure 7 (A) Antral mucosoid cultures infected for 72 hours at MOI 50 labelled with antibody against CagA showing groups of bacteria that have not yet adhered to the cells $\left({ }^{*}\right)$ or adherent to the surface $\left({ }^{* *}\right)$. (B) Western blot analysis of phosphorylated CagA detected with an antibody against PY99 in cell lysates from non-infected cells (left lane), antral mucosoid cultures infected with P12 at MOI 100 for 72 hours (middle lane) and the same cells seeded as planar monolayers on collagen-coated plastic infected for 18 hours at MOI 25 (right lane) (C) left panel: EM of an antral mucosoid culture showing the interaction between the microvilli and foci of coccoid shaped bacteria 24 hours after infection: right panel: a helical bacterium adhering on the apical surface 6 hours after infection. P12-GFP MOI=100. (D) Paraffin section of a mucosoid culture infected with P12 at M0I 50 for 72 hours. The bright layer indicates the cell layer. The bacteria are mostly bound to a continuous mucus layer that only a few have managed to infiltrate (arrowheads). (E) Mucus was harvested from mucosoid cultures infected with P12 at MOI 100 or uninfected controls, centrifuged to remove bacteria and used to plate the kanamycin resistant isogenic strain P12-GFP for 1 and 2 hours. The sample was plated on kanamycin plates and colonies counted relative to the number of bacteria plated. Results represent percent of colonies compared with the input $+/$-SD of three mucus samples. EM, electron micrograph; H. pylori, Helicobacter pylori; PY99, phosphorylated tyrosine.

focalised and individual bacteria leaving the foci might succumb when exposed to the mucus.

\section{Long-term infection of gastric mucosoid cultures with $\mathrm{H}$. pylori}

To model long-term infection with $H$. pylori, we infected mucosoid cultures for 24 hours at MOI 100 and washed the surface with phosphate buffered saline (PBS) after the first day to remove free-swimming bacteria. To monitor the dynamics of the bacteria, we used an isogenic P12 strain expressing GFP. Infections were terminated after 4 weeks, which far exceeds any other infection model, especially when using extracellular bacteria. The accumulating mucus containing bacteria was removed regularly but only sporadically formed colonies when transferred to agar (not shown), probably due to the high toxicity of the mucus. Confocal images of long-term infected monolayers show that the bacteria are highly condensed in clusters of $\sim 5 \mu \mathrm{m}$ diameter (figure 8A). High-magnification images show that individual 
A

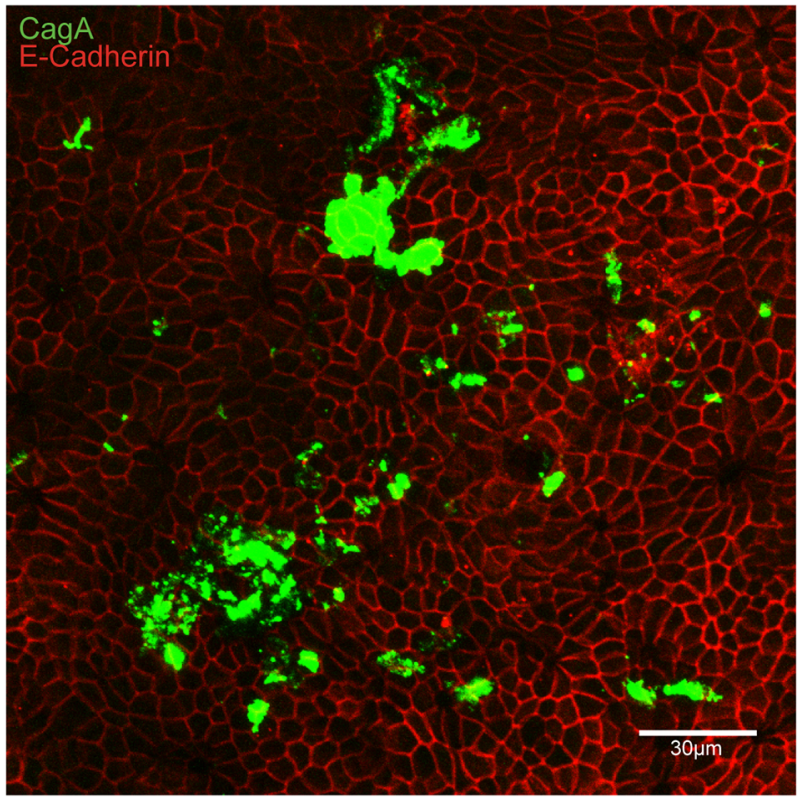

C

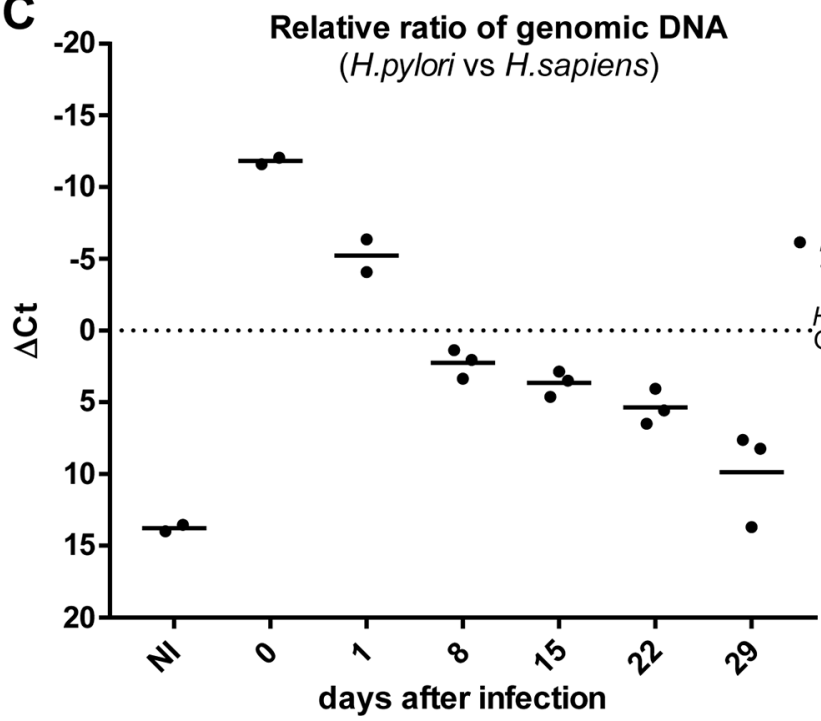

B

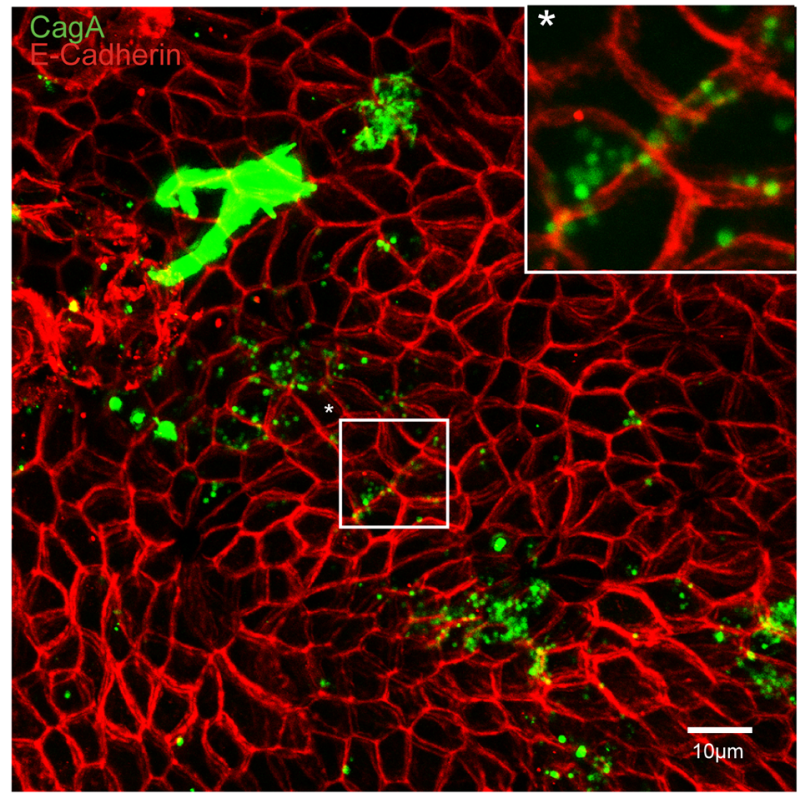

D

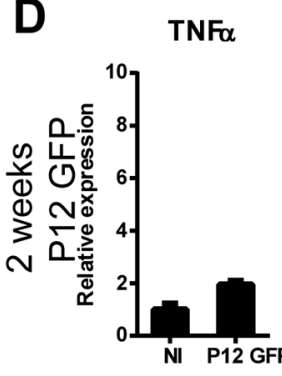

IL-8

MUC5AC
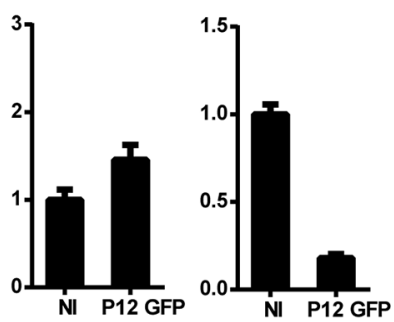

E

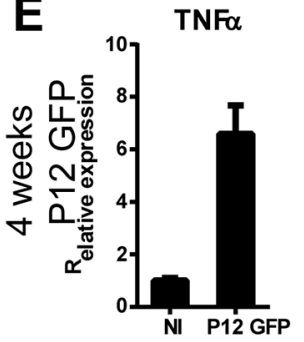

Figure 8 Antral mucosoid cultures were infected with the isogenic $H$. pylori strain P12-GFP at MOI 100. Twenty-four hours later, the surface was rinsed twice with PBS and mucus removed every 3 to 4 days. (A) After 2 weeks, filters were fixed and labelled with antibodies against cagA and E-cadherin followed by whole mount IF. (B) Higher magnification image and detail $\left({ }^{*}\right)$ enlarged in top right corner of the same infection as in (A). (C) The relative amount of P12-GFP to the host cells was calculated by qPCR comparing the abundance of the $16 \mathrm{~s}$ ribosomal DNA sequence versus the one of human GAPDH. (D, E) Expression of $T N F-\alpha, I L-8$ and MUC5AC was analysed by qRT-PCR after 2 and 4 weeks of infection and normalised to non-infected control. Day - 0 DNA was collected directly after application of bacteria; day-1 DNA was collected after washing with PBS. $\triangle$ $\mathrm{Ct}$ - difference between the $\mathrm{Ct}$ of each gene compared with GAPDH, the horizontal sign is the average of the values represented by the dots, each dot is one replicate. GAPDH, glyceraldehyde 3-phosphate dehydrogenase; IL, interleukin; NI, non-infected; PBS, phosphate buffered saline; qPCR, quantitative PCR; TNF- $\alpha$, tumour necrosis factor alpha.

bacteria outside of the clusters have a coccoid shape, reminiscent of the action of defensins ${ }^{31}$ (figure $8 \mathrm{~B}$ and enlargement). The relative number of bacterial versus human cells was monitored every week by qPCR of the respective genomic DNA (figure 8C). At day 0, bacteria were lysed immediately. At day 1, the lysis was carried out after washing with PBS, and every week thereafter mucus was removed first. The number of bacteria diminished over time and was undetectable at 4 weeks (figure $8 \mathrm{C}$ ). However, mRNA levels of TNF- $\alpha$ and $I L-8$ were only slightly increased at 2 weeks (figure $8 D$ ) but became markedly elevated by 4 weeks of infection (figure 8E). Expression of MUC5AC mRNA was drastically reduced at both time points (figure $8 \mathrm{D}, \mathrm{E}$ ). We conclude that our system is able to model the effect of long-term infection and shows persistent inflammation.

\section{DISCUSSION}

Using the novel mucosoid culture system of gastric epithelium, we provide interesting insight into various aspects of the pathogen-host tissue interplay, reminiscent of the authentic in 
vivo condition. Owing to the striking degree of polarisation and the functional integrity of the epithelium in conjunction with a greatly reduced passaging frequency, we achieved full differentiation into all antral phenotypes, which has not been reported before in pure adult epithelial cultures. Alterations of Wnt-signalling intensity was found to control the differentiation of basal into foveolar cell types. Apical mucus secretion constitutes a protective shield against bacteria, preventing excessive host cell death and, in turn, providing a condition of homeostatic infection with $H$. pylori. Probing the effects of stromal cells from the lamina propria surrounding the gastric glands showed that they induce differentiation through the action of secreted Wnt inhibitors. Future studies with this model will thus enable detailed insight into the signalling routes between the epithelium and the stroma and how they are affected by bacterial infection.

The profound effects resulting from alterations in the microenvironmental signalling are illustrated by the surprising ease with which cultures are transformed from a MUC6 ${ }^{\text {hi }}$ gland base phenotype towards a MUC5AChi foveolar phenotype on withdrawal of Wnt3A and RSPO1. Infection of these distinct monolayer phenotypes indicates that activation of the NF- $\mathrm{KB}$ pathway is substantially higher at the gland base than in the foveolae. This phenotype was evidenced by the upregulation of p65 target genes in the basal-type cultures, including the inflammatory mediators LTB, IL-8, CXCL1-3, CCL20, IL-23A and TNF- $\alpha$ previously found to be overexpressed H. pylori-infected patients. ${ }^{32-34}$ The foveolar lineage, on the other hand, was also less sensitive to the macrophage-derived proinflammatory cytokine IL-1 $\beta$. Together, these observations support the notion that superficial infections with $H$. pylori on the gland surface provoke only minor immunological responses, likely explaining the absence of apparent symptoms in healthy $H$. pylori carriers. Future studies with gastric mucosoid cultures will help reveal to which extent the recently described $H$. pylori type 4 secretion system (cag PAI)-mediated translocation of the heptose 1,7-bisphosphate (HBP) effector molecule ${ }^{35} 36$ accounts for NF- $\mathrm{KB}$ induction in this highly authentic scenario of epithelial polarisation and function.

Another intriguing outcome is the apparent link between the NF- $\mathrm{KB}$ and Wnt pathways. Foveolar-type cells emerge in the absence of Wnt signalling or expression of Wnt target genes. Indeed, the main mediator of Wnt signalling, $\beta$-catenin, has previously been shown to regulate inflammation via cross-talk with the NF- $\mathrm{KB}$ signalling pathway, ${ }^{37}$ corroborating the notion that active Wnt signalling may confer competence of the epithelial compartment to respond to inflammatory signals. With the new infection model, we anticipate the generation of novel insights into the coordination of these two pathways in the gastric gland during infection. Notably, this approach could provide explanations for the link between inflammation and altered homeostasis of the stem cell compartment during chronic $H$. pylori infection, and how it may lead to malignant transformation.

H. pylori can enter deep into the glands and interact with the stem cell compartment where it triggers hyperplasia ${ }^{11}$ and inflammation. Immune cells such as macrophages, T lymphocytes and $\mathrm{B}$ cells then infiltrate the mucosa and target the epithelium, ${ }^{38}$ exacerbating inflammation. Moreover $H$. pylori can destabilise the host genome by inducing DNA doublestrand breaks and shifting repair mechanisms towards errorprone non-homologous end joining. ${ }^{39}{ }^{40}$ This combination of a chronic inflammatory, proregenerative environment and the marked genotoxic effect exerted by the bacteria might promote the development of neoplasia. Gastric mucosoid cultures offer a unique tool for investigating early carcinogenic events, as they enable an unprecedented prolongation of the infection period, coupled with the ability to expose the epithelium to a minimal cytokine milieu in the basal compartment to mimic a chronic inflammatory environment.

To colonise the epithelial surface, the bacteria have to overcome the gel-like gastric mucus lining propelled by their flagella. ${ }^{41}$ We observed that only a few bacteria managed to traverse the mucus to establish sparse bacterial colonies. Two days after inoculation of the cultures, most bacteria are still located on the surface of the mucus, suggesting that variations in bacterial fitness and maintenance of an effective mucus barrier play a role in controlling the pathogens' fate. Unlike the mucosoid cultures, organoid cultures are typically grown in Matrigel, which is stabilised by antibiotics, potentially affecting bacterial fitness. It has been shown that mucus secretion plays a central role in protecting the epithelium in other regions of the GI tract. ${ }^{42}{ }^{43}$ We are only just beginning to appreciate the important role played by mucus in the GI tract, for example as a site of immune defence, and the mucosoid culture system allows investigation of the mucus within the culture and produces abundant mucus that can be harvested for further analysis.

Our model thus lays the grounds for studying the determinants and effects of chronic $H$. pylori infection in vitro, as well as the effect of mucins and mucin modifications on commensal and pathogenic bacteria. The procedure, which works equally well for establishing mucosoids from the gastric corpus (data not shown), will likely be transferable to other columnar epithelia from human and other mammalian species and might also prove to be useful as a drug screening tool with an impact on future personalised medicine.

\section{MATERIALS AND METHODS Human tissue material, gastric gland isolation and spheroid culture}

Gastric tissue samples were provided by the Center of Bariatric and Metabolic Surgery, Charité University Medicine and by the Center for Obesity and Metabolic Medicine, Helios Klinikum, (both in Berlin, Germany) with prior approval of the ethics committee of the Charité University Medicine, Berlin (EA1/129/12). Pseudonymised samples (see online supplementary table 2) were obtained from a total of 12 patients negative for $H$. pylori undergoing stomach sleeve resection and processed as described previously for organoid cultures. ${ }^{14}$ Briefly, tissue was washed in PBS before removing fat and connective tissue. It was then cut into $\sim 1 \mathrm{~mm}^{2}$ pieces and washed in cold PBS until the supernatant was clear $(8-10$ times), followed by a $30 \mathrm{~min}$ incubation in chelating solution (5.6 mM Na2HPO4/8.0 mM KH2PO4/96.2 mM NaCl/1.6 mM $\mathrm{KCl} / 43.4 \mathrm{mM}$ sucrose $/ 54.9 \mathrm{mM}$ D-sorbitol/ $/ 0.5 \mathrm{mM}$ DL-dithiothreitol $/ 2 \mathrm{mM}$ EDTA in $\mathrm{H}_{2} \mathrm{O}$ ) at $37^{\circ} \mathrm{C}$ on a shaking platform. Tissue fragments were left to settle, transferred to a petri dish and glands isolated by applying gentle pressure with a glass slide, before resuspending in medium containing $10 \%$ heat-inactivated fetal calf serum (Biochrom). After transfer to a Falcon tube, larger fragments were removed by leaving them to settle for 1 min before transferring the supernatant, containing most of the isolated glands, to a new tube. After washing, the solution was centrifuged for $5 \mathrm{~min}$ at $250 \mathrm{~g}$, resuspended in advanced Dulbecco's modified Eagle medium/F12 (Invitrogen) and triturated with a flame-polished glass pipette until only single cells and very small clumps remained. The number of cells was estimated using a haemocytometer, and resuspended in an appropriate 
volume of culture medium to generate mucosoid cultures or initiate organoid cultures.

\section{Generation of gastric mucosoid cultures}

Around 200000 to 250000 cells derived either directly from freshly isolated glands or from organoids were seeded in $200 \mu \mathrm{L}$ culture medium into collagen-coated (Gibco A10644-01, $15 \mu \mathrm{g}$ / $\mathrm{cm}^{2}$ ) transwell inserts (Millipore PIHP01250) placed in a 24-well plate at $37^{\circ} \mathrm{C}, 5 \% \mathrm{CO}_{2}$ in a humidified incubator. The space between filter and well was filled with $400 \mu \mathrm{L}$ culture medium (see online supplementary table 2 ). On day 3 , the medium overlying the cells was removed from the well insert to start the ALI culture. Subsequently the $500 \mu \mathrm{L}$ medium below the filter was replaced twice a week.

\section{Infection with H. pylori}

H. pylori P12 (strain collection no. P511) and isogenic P12-GFP strains (strain collection no. P421) were grown and collected for infection as described before. ${ }^{14}$ Bacterial number was determined by measuring the optical density at $550 \mathrm{~nm}$. Infection was carried out in $30 \mu \mathrm{L}$ of PBS on top of the filters for 6 hours to 3 days. Long-term infection (2 and 4 weeks) was performed at MOI 100 for 1 day followed by washing with PBS and removal of excess mucus at every medium change.

\section{Antibodies and primers}

See online supplementary tables 4 and 5 .

Acknowledgements The authors would like to thank Dagmar Frahm for support with the cultures, Hans-Joachim Mollenkopf and Ina Wagner for support with the microarray experiments, Diane Schad for help with figure preparation and Rike Zietlow for editing the manuscript. We thank the shared mass spectrometry facility of the Charite for support in acquisition of the data.

Contributor FB: study design, experimental design, acquisition of data, analysis and interpretation of data, drafting of the manuscript, statistical analysis. SW, Al-M and PM: experimental design, acquisition of data, and analysis and interpretation of data. GS: acquisition of data. CG: electron microscopy. HB: bioinformatics support, statistical analysis. MS: mass spectrometry. CD and JO: provision of materials. TFM: study concept and design, critical revision of the manuscript, obtained funding and study supervision.

Competing interests None declared.

Patient consent Obtained.

Ethics approval Ethics committee of the Charité University Medicine, Berlin (EA1/129/12)

Provenance and peer review Not commissioned; externally peer reviewed.

Open access This is an open access article distributed in accordance with the Creative Commons Attribution Non Commercial (CC BY-NC 4.0) license, which permits others to distribute, remix, adapt, build upon this work non-commercially, and license their derivative works on different terms, provided the original work is properly cited and the use is non-commercial. See: http://creativecommons.org/ licenses/by-nc/4.0/

(c) Article author(s) (or their employer(s) unless otherwise stated in the text of the article) 2019. All rights reserved. No commercial use is permitted unless otherwise expressly granted.

\section{REFERENCES}

1 Blaser MJ, Perez-Perez GI, Kleanthous H, et al. Infection with Helicobacter pylori strains possessing cagA is associated with an increased risk of developing adenocarcinoma of the stomach. Cancer Res 1995;55:2111-5.

2 Parsonnet J, Friedman GD, Orentreich N, et al. Risk for gastric cancer in people with CagA positive or CagA negative Helicobacter pylori infection. Gut 1997;40:297-301.

3 Hatakeyama M. Helicobacter pylori CagA and gastric cancer: a paradigm for hit-andrun carcinogenesis. Cell Host Microbe 2014;15:306-16.

4 Correa P, Piazuelo MB. The gastric precancerous cascade. J Dig Dis 2012;13:2-9.

5 Rakoff-Nahoum S. Why cancer and inflammation? Yale J Biol Med 2006;79(3-

4):123-30.

6 Coussens LM, Werb Z. Inflammation and cancer. Nature 2002;420:860-7.
7 Huang JY, Sweeney EG, Sigal M, et al. Chemodetection and destruction of host urea allows Helicobacter pylori to locate the epithelium. Cell Host Microbe 2015; 18:147-56.

8 Arwert EN, Hoste E, Watt FM, et al. wound healing and cancer. Nat Rev Cancer 2012;12:170-80.

9 Mahdavi J, Sondén B, Hurtig M, et al. Helicobacter pylori SabA adhesin in persistent infection and chronic inflammation. Science 2002;297:573-8.

10 Howitt MR, Lee JY, Lertsethtakarn P, et al. ChePep controls Helicobacter pylori Infection of the gastric glands and chemotaxis in the Epsilonproteobacteria. MBio 2011;2:e00098-11.

11 Sigal M, Rothenberg ME, Logan CY, et al. Helicobacter pylori activates and expands Lgr5(+) stem cells through direct colonization of the gastric glands. Gastroenterology 2015;148:1392-404.

12 Barker N, Huch M, Kujala P, et al. Lgr5(+ve) stem cells drive self-renewal in the stomach and build long-lived gastric units in vitro. Cell Stem Cell 2010;6:25-36.

13 Ootani A, Li X, Sangiorgi E, et al. Sustained in vitro intestinal epithelial culture within a Wnt-dependent stem cell niche. Nat Med 2009;15:701-6.

14 Schlaermann $\mathrm{P}$, Toelle $\mathrm{B}$, Berger $\mathrm{H}$, et al. A novel human gastric primary cell culture system for modelling Helicobacter pylori infection in vitro. Gut 2016;65:202-13.

15 Bartfeld S, Bayram T, van de Wetering M, et al. In vitro expansion of human gastric epithelial stem cells and their responses to bacterial infection. Gastroenterology 2015;148:126-36.

16 de Lau W, Peng WC, Gros P, et al. The R-spondin/Lgr5/Rnf43 module: regulator of Wnt signal strength. Genes Dev 2014;28:305-16.

17 Katano T, Ootani A, Mizoshita T, et al. Gastric mesenchymal myofibroblasts maintain stem cell activity and proliferation of murine gastric epithelium in vitro. Am J Pathol 2015; 185:798-807.

18 Ootani A, Toda S, Fujimoto K, et al. Foveolar differentiation of mouse gastric mucosa in vitro. Am J Pathol 2003;162:1905-12.

19 Sigal M, Logan CY, Kapalczynska M, et al. Stromal R-spondin orchestrates gastric epithelial stem cells and gland homeostasis. Nature 2017;548:451-5.

20 Bartfeld S, Clevers H. Organoids as model for infectious diseases: culture of human and murine stomach organoids and microinjection of Helicobacter pylori. J Vis Exp 2015. doi: 10.3791/53359. [Epub ahead of print 12 Nov 2015].

21 Bartfeld S. Modeling infectious diseases and host-microbe interactions in gastrointestinal organoids. Dev Biol 2016;420:262-70.

22 Butor C, Davoust J. Apical to basolateral surface area ratio and polarity of MDCK cells grown on different supports. Exp Cell Res 1992;203:115-27.

23 Sato T, Vries RG, Snippert HJ, et al. Single Lgr5 stem cells build crypt-villus structures in vitro without a mesenchymal niche. Nature 2009;459:262-5.

24 Khurana SS, Riehl TE, Moore BD, et al. The hyaluronic acid receptor CD44 coordinates normal and metaplastic gastric epithelial progenitor cell proliferation. J Biol Chem 2013;288:16085-97.

25 Srinivasan $B$, Kolli AR, Esch MB, et al. TEER measurement techniques for in vitro barrier model systems. J Lab Autom 2015;20:107-26.

26 Gudipaty SA, Rosenblatt J. Epithelial cell extrusion: pathways and pathologies. Semin Cell Dev Biol 2017;67:132-40.

27 Madara JL. Maintenance of the macromolecular barrier at cell extrusion sites in intestinal epithelium: physiological rearrangement of tight junctions. J Membr Biol 1990:116:177-84.

28 Choi E, Roland JT, Barlow BJ, et al. Cell lineage distribution atlas of the human stomach reveals heterogeneous gland populations in the gastric antrum. Gut 2014;63:1711-20.

29 Nordman H, Davies JR, Lindell G, et al. Gastric MUC5AC and MUC6 are large oligomeric mucins that differ in size, glycosylation and tissue distribution. Biochem J 2002;364(Pt 1):191-200.

30 Babu SD, Jayanthi V, Devaraj N, et al. Expression profile of mucins (MUC2, MUC5AC and MUC6) in Helicobacter pylori infected pre-neoplastic and neoplastic human gastric epithelium. Mol Cancer 2006;5:10.

31 Bauer B, Pang E, Holland C, et al. The Helicobacter pylori virulence effector CagA abrogates human $\beta$-defensin 3 expression via inactivation of EGFR signaling. Cell Host Microbe 2012;11:576-86.

32 Cook KW, Letley DP, Ingram RJ, et al. CCL20/CCR6-mediated migration of regulatory $T$ cells to the Helicobacter pylori-infected human gastric mucosa. Gut 2014;63:1550-9.

33 Hor YT, Voon DC, Koo JK, et al. A role for RUNX3 in inflammation-induced expression of IL23A in gastric epithelial cells. Cell Rep 2014;8:50-8.

34 Mejías-Luque $R$, Zöller J, Anderl F, et al. Lymphotoxin $\beta$ receptor signalling executes Helicobacter pylori-driven gastric inflammation in a T4SS-dependent manner. Gut 2017;66.

35 Gall A, Gaudet RG, Gray-Owen SD, et al. TIFA signaling in gastric epithelial cells initiates the cag type 4 secretion system-dependent innate immune response to Helicobacter pylori Infection. MBio 2017;8:e01168-17.

36 Zimmermann S, Pfannkuch L, Al-Zeer MA, et al. ALPK1- and TIFA-dependent innate immune response triggered by the Helicobacter pylori type IV secretion system. Cell Rep 2017;20:2384-95.

$37 \mathrm{Ma} \mathrm{B}$, Hottiger MO. Crosstalk between Wnt/ $\beta$-catenin and NF- $\kappa B$ signaling pathway during inflammation. Front Immunol 2016;7:378 
38 Neumann L, Mueller M, Moos V, et al. Mucosal inducible NO synthaseproducing IgA+ plasma cells in Helicobacter pylori-infected patients. J Immunol 2016;197:1801-8.

39 Hartung ML, Gruber DC, Koch KN, et al. H. pylori-induced DNA strand breaks are introduced by nucleotide excision repair endonucleases and promote NF- $\kappa B$ target gene expression. Cell Rep 2015;13:70-9.

40 Koeppel M, Garcia-Alcalde F, Glowinski F, et al. Helicobacter pylori infection causes characteristic DNA damage patterns in human cells. Cell Rep 2015;11:1703-13.
41 Bansil R, Celli JP, Hardcastle JM, et al. The influence of mucus microstructure and rheology in Helicobacter pylori infection. Front Immunol 2013;4:310.

42 Birchenough GM, Nyström EE, Johansson ME, et al. A sentinel goblet cell guards the colonic crypt by triggering Nlrp6-dependent Muc2 secretion. Science 2016;352:1535-42.

43 Propheter DC, Chara AL, Harris TA, et al. Resistin-like molecule $\beta$ is a bactericidal protein that promotes spatial segregation of the microbiota and the colonic epithelium. Proc Natl Acad Sci U S A 2017;114:11027-33. 